University of Zurich

Department of Economics

Working Paper Series

ISSN 1664-7041 (print)

ISSN1664-705X(online)

Working Paper No. 18

\title{
On Uniqueness and Stability of symmetric equilibria in differentiable symmetric games
}

Andreas Hefti

May 2011 


\title{
On Uniqueness and Stability of symmetric equilibria in differentiable symmetric games*
}

\author{
Andreas Hefti ${ }^{\dagger}$
}

February 2011

\begin{abstract}
Higher-dimensional symmetric games become of more and more importance for applied micro- and macroeconomic research. Standard approaches to uniqueness of equilibria have the drawback that they are restrictive or not easy to evaluate analytically. In this paper I provide some general but comparably simple tools to verify whether a symmetric game has a unique symmetric equilibrium or not. I distinguish between the possibility of multiple symmetric equilibria and asymmetric equilibria which may be economically interesting and is useful to gain further insights into the causes of asymmetric equilibria in symmetric games with higher-dimensional strategy spaces. Moreover, symmetric games may be used to derive some properties of the equilibrium set of certain asymmetric versions of the symmetric game. I further use my approach to discuss the relationship between stability and (in)existence of multiple symmetric equilibria. While there is an equivalence between stability, inexistence of multiple symmetric equilibria and the unimportance of strategic effects for the comparative statics, this relationship breaks down in higher dimensions. Stability under symmetric adjustments is a minimum requirement of a symmetric equilibrium for reasonable comparative statics of symmetric changes. Finally, I present an alternative condition for a symmetric equilibrium to be a local contraction which is more general than the conventional approach of diagonal dominance and yet simpler to evaluate than the eigenvalue condition of continuous adjustment processes.
\end{abstract}

Keywords: Symmetric Games, Nash Equilibrium, Uniqueness, Stability JEL Classification: C72

${ }^{*}$ I wish to thank Diethard Klatte, Armin Schmutzler, Nick Netzer and Josef Falkinger for valuable comments on the subject of this paper. Janette Garcia provided excellent research assistance.

${ }^{\dagger}$ University of Zurich, Socioeconomic Institute, Zürichbergstrasse 14, CH-8032 Zurich. E-mail: a.hefti@wwi.uzh.ch 


\section{Introduction}

In the micro- and macroeconomic literature symmetric games usually are the simplest approach to investigate the equilibrium response of the players involved to some exogenous change. Often, the equilibrium properties of the symmetric game is of self-interest and sometimes conditions that assert properties as uniqueness or stability of the equilibrium in the symmetric game provide further insights about the properties of the equilibrium set in asymmetric variations of the game. Unfortunately, standard methods to investigate the properties of the equilibrium set of a symmetric game are either very restrictive (contraction or univalence approach) and generally difficult to evaluate analytically because, especially in higher dimensions, large Jacobian matrices are involved.

In this paper I develop comparably simple conditions that assert whether a differentiable symmetric game has a unique equilibrium. My approach to uniqueness is different from the literature as I distinguish between multiple symmetric equilibria and asymmetric equilibria. By taking explicit advantage of the symmetry in the game I derive two criteria that, if both are satisfied, assert that only one equilibrium, the symmetric equilibrium, exists. One criterion rules out the possibility of multiple symmetric equilibria and the other criterion rules out the possibility of asymmetric equilibria. The method presented in this paper shows that the complexity of examining uniqueness in a general $N$-player game can be reduced to the case of a two-player game. Moreover, the index theorem, commonly accepted as the most general approach to uniqueness, is only applicable under the assumption of severe boundary conditions - a requirement that can be weakened as this paper reveals. Moreover, by examining the equilibrium set of a symmetric game we can derive certain properties of the equilibrium set of asymmetric variations of the game. This insight is substantial as the symmetric game usually is a lot easier to work with.

The paper is structured as follows. After building up the required notation I derive and discuss the two criteria, that together assert uniqueness of the symmetric equilibrium in case of one- and two-dimensional strategy spaces ${ }^{1}$. As an interesting result we see that in two-dimensional games with at most weakly decreasing direct responses there can never be asymmetric equilibria if the game has an increasing and a decreasing cross-over response or at least one cross-over response is constant. Despite the comparably high simplicity, my approach still is very general as the two conditions, when applied to the Cournot game, also produce the two main conditions of

\footnotetext{
${ }^{1}$ I also illustrate that the method extends in a straightforward way to higher dimensional games.
} 
uniqueness that have been recognized by the literature. My approach enhances these conditions with a new interpretation. Further, I show that there is an intimate connection between the inexistence of asymmetric equilibria in the symmetric game and whether or not we can expect an asymmetric variation of the game to produce equilibria with a strategy-ordering that reflects asymmetry (e.g. the firm with cheaper production costs will set a lower price in any equilibrium). In section $4 \mathrm{I}$ expose the relationship between stability of a symmetric equilibrium and multiplicity of symmetric equilibria. Generally, the existence of multiple symmetric equilibria, rather than the existence of asymmetric equilibria, is problematic for stability and comparative statics. While stability under symmetric adjustments, inexistence of multiple symmetric equilibria and the unimportance of strategic effects for the sign of the comparative statics are the same properties of a one-dimensional game this equivalence breaks down in higher dimensions. Nevertheless, a restricted version of myopic stability, stability under symmetric adjustments, is necessary to obtain reasonable comparative statics of symmetric equilibria. Finally, I derive a condition that asserts whether a symmetric equilibrium is a local contraction, which is simple to check, and yet more general than the usual requirement of a dominant diagonal.

\section{Existence of symmetric equilibria}

In this section I build up the notation and then derive a sufficient condition for the existence of a symmetric equilibrium.

\section{$2.1 \quad$ Notation and assumptions}

The notation follows Topkis (Topkis (1998)). Consider a non-cooperative game of $2 \leq N<$ $\infty$ identical players. Players are indexed by a number $1, \ldots, N$ and all players have the same strategy space. Throughout the paper strategies are defined to be pure strategies. Let $x_{g} \equiv$ $\left(x_{g 1}, \ldots, x_{g k}\right) \in S(k)$ denote a feasible strategy of player $g$ where $S(k) \subset \mathbb{R}_{+}^{k}$ denotes the strategy space of the player. Let $S \equiv S(k)$ be the product space $S=\times_{i=1}^{k} S_{i}$ where $S_{i}=\left[0, \bar{S}_{i}\right] \subset \mathbb{R}_{+}$ with $\bar{S}_{i}>0$ for all $i=1, \ldots, k$. Thus $S$ is a nonempty, compact and convex subset of an Euclidian space. Further $x=\left(x_{1}, \ldots, x_{N}\right) \in S^{N} \subset \mathbb{R}_{+}^{k N}$ denotes a strategy profile and $S^{N}=S \times \ldots \times S$ also is compact and convex. For a player $g$ and a given strategy profile $x$ the vector $x_{-g} \in S^{N-1}$ denotes the vector of strategies of all $(N-1)$ players except player $g$. Sometimes I simply write 
$x=\left(x_{g}, x_{-g}\right)$. The payoff function of player $g$ is a real-valued and differentiable function $\Pi^{g}(x)$ defined on $S^{N}$. Following Dasgupta and Maskin symmetry means permutation-invariance of the payoff functions (Dasgupta and Maskin (1986)):

$$
\Pi^{g}\left(x_{1}, \ldots, x_{g}, \ldots, x_{N}\right)=\Pi^{\sigma(g)}\left(x_{\sigma(1)}, \ldots, x_{\sigma(g)}, \ldots, x_{\sigma(N)}\right)
$$

for $g=1, \ldots, N$ where $\sigma$ is a permutation of the set $\{1, \ldots, N\}$. This especially implies that

$$
\Pi^{g}\left(x_{1}, \ldots, x_{g}, \ldots, x_{N}\right)=\Pi^{g}\left(x_{\mu(1)}, \ldots, x_{g}, \ldots, x_{\mu(N)}\right)
$$

for $g=1, \ldots, N$ where $\mu$ is a permutation of $\{1, \ldots, N\} \backslash\{g\}$. For simple reference I set

$$
\Pi\left(x_{g}, x_{-g}\right) \equiv \Pi^{g}\left(x_{1}, \ldots, x_{g}, \ldots, x_{N}\right) \quad g=1, \ldots, N
$$

The non-cooperative $k$-dimensional symmetric game is the triple $\left(N, S(k)^{N}, \Pi\right)$. Throughout this paper I shall maintain the following assumption on $\Pi$ :

Assumption 1 For $g \in\{1, \ldots, N\}$ the payoff function $\Pi\left(x_{g}, x_{-g}\right) \in C^{2}\left(S^{N}, \mathbb{R}\right)$ satisfies (1) and is strictly quasiconcave in $x_{g} \in S$ for any $x_{-g} \in S^{(N-1)}$.

The property of permutation-invariance implies ${ }^{2}$

$$
\frac{\partial \Pi^{g}\left(x_{1}, \ldots, x_{g}, \ldots, x_{N}\right)}{\partial x_{g}}=\frac{\partial \Pi^{g}\left(x_{\mu(1)}, \ldots, x_{g}, \ldots, x_{\mu(N)}\right)}{\partial x_{g}}
$$

For simple reference I set

$$
\Pi_{i}\left(x_{g}, x_{-g}\right) \equiv \frac{\partial \Pi^{g}\left(x_{1}, \ldots, x_{g}, \ldots, x_{N}\right)}{\partial x_{g i}} \quad 1 \leq i \leq k, \quad 1 \leq g \leq N
$$

In a non-cooperative game every player $g$ solves

$$
\max _{x_{g} \in S} \Pi\left(x_{g}, x_{-g}\right)
$$

\footnotetext{
${ }^{2}$ Note that the following expression is a vector derivative.
} 
I denote the best response function of player $g$ by

$$
\varphi^{g}: S^{N-1} \rightarrow S \quad, x_{-g} \mapsto \varphi^{g}\left(x_{-g}\right)
$$

and use $\varphi\left(x_{-g}\right) \equiv \varphi^{g}\left(x_{-g}\right)$ for simple reference. Similarly,

$$
\varphi_{i}^{g}: S^{N-1} \rightarrow S_{i} \quad, x_{-g} \mapsto \varphi_{i}^{g}\left(x_{-g}\right)
$$

is the $i$-th component $(1 \leq i \leq k)$ of $\varphi^{g}$ and I set $\varphi_{i}\left(x_{-g}\right) \equiv \varphi_{i}^{g}\left(x_{-g}\right)$.

To find a symmetric equilibrium in specific symmetric games usually a simplified approach, the symmetric opponents form approach (SOFA), is used (e.g. Dixit (1986), p. 116 or Grossman and Shapiro (1984) or Salop (1979)). The SOFA takes player $g=1$ and sets $x_{-1}=\bar{x}_{-1} \equiv(\bar{x}, \ldots, \bar{x})$ where $\bar{x} \in S$ and $\tilde{\Pi}\left(x_{1}, \bar{x}\right) \equiv \Pi^{1}\left(x_{1}, \bar{x}_{-1}\right)$ and then solves

$$
\max _{x_{1} \in S} \tilde{\Pi}\left(x_{1}, \bar{x}\right)
$$

Let $\tilde{\varphi}(\bar{x}) \equiv \varphi\left(\bar{x}_{-1}\right) \in S$ denote the set of maximisiers of (3). A symmetric equilibrium of $\left(N, S^{N}, \Pi\right)$ then is a point $x^{*} \in S^{N}$ with $x_{1}^{*}=\ldots=x_{N}^{*}$ and $x_{1}^{*} \in \tilde{\varphi}\left(x_{1}^{*}\right)$.

\subsection{An existence result}

It is well-known that the set of all pure-strategy equilibrium points of a non-cooperative game is identical to the set of fixed points of the joint best response correspondence (see, e.g. Topkis (1998), p. 179). The following proposition is easy to prove.

Proposition 1 Assumption 1 implies the following two facts for the symmetric game $\left(N, S^{N}, \Pi\right)$ :

(i) There exists a continuous joint best-response function

$$
\phi: S^{N} \rightarrow S^{N} \quad, x \mapsto\left(\begin{array}{c}
\varphi\left(x_{-1}\right) \\
\vdots \\
\varphi\left(x_{-N}\right)
\end{array}\right)
$$

(ii) $\left(N, S^{N}, \Pi\right)$ has a symmetric equilibrium $x^{*} \in S^{N}$ 
Proof: Appendix (6.2)

A question frequently asked is, when do only interior equilibria exist. Let

$$
\nabla F(x) \equiv\left(\begin{array}{c}
\nabla \Pi^{1}\left(x_{1}, \ldots, x_{N}\right) \\
\nabla \Pi^{2}\left(x_{1}, \ldots, x_{N}\right) \\
\vdots \\
\nabla \Pi^{N}\left(x_{1}, \ldots, x_{N}\right)
\end{array}\right)
$$

denote the pseudogradient (a $N k$ vector; Rosen $(1965)$ ) of $\left(N, S^{N}, \Pi\right.$ ) at $x$ where

$\nabla \Pi^{g}\left(x_{1}, \ldots, x_{N}\right)$ for $g=1, \ldots, N$ is the gradient (a $k$-vector) of the payoff function of player $g$ with respect to $x_{g}$. Let $\hat{E} \equiv\left\{x \in S^{N}: \phi(x)=x\right\}$ denote the set of all fixed points of $\phi$. The set $E$ of all interior equilibria (which might be empty) is

$$
E \equiv\left\{x \in \operatorname{Int}\left(S^{N}\right): \nabla F(x)=0\right\}
$$

where $\operatorname{Int}\left(S^{N}\right)$ denotes the interior of $S^{N}$. Let $J(x)$ denote the Jacobian (a $N k \times N k$ matrix) of $\nabla F(x)$ with respect to $x$. The following assumption asserts that only interior equilibria exist.

Assumption 2 (Boundary condition) The gradient field

$$
\nabla F: S^{N} \rightarrow \mathbb{R}^{N k} \quad, x \mapsto \nabla F(x)
$$

points into the interior of $S^{N}$ at all boundary points.

Proposition 2 The following two properties characterise the set of equilibria of $\left(N, S^{N}, \Pi\right)$

i) Under assumption 2 only interior equilibria exist: $\hat{E}=E$

ii) If all equilibrium points are regular, i.e. the Jacobian $J(x)$ has $\operatorname{Det}(J(x)) \neq 0$ for all $x \in E$, then $E$ is a finite set.

Proof: Appendix (6.3)

Intuitively, i) is true because if assumption 2 holds but there also were a boundary equilibrium this would contradict the Kuhn-Tucker necessary and sufficient conditions. ii) holds because the 
regularity condition implies that all equilibrium points are locally isolated and a compact and "discrete" set must be finite. Note that assumption 2 is sufficient (but not necessary) for ruling out boundary equilibria.

When dealing with asymmetric equilibria I will require the best response function $\varphi\left(x_{-g}\right)$ to be differentiable at certain points. As the following corollary states, assumption 1 asserts that $\varphi\left(x_{-g}\right)$ is differentiable in $x_{-g}$ if $\varphi\left(x_{-g}\right) \in \operatorname{Int}(S)$.

Corollary 1 If $\varphi\left(x_{-g}\right) \in \operatorname{Int}(S)$ then $\varphi\left(x_{-g}\right)$ is differentiable at $x_{-g}$.

Proof: Appendix (6.4)

\section{Uniqueness}

This section first summarises the main two approaches to uniqueness: the univalence approach and the index approach. Then I develop the condition which excludes asymmetric equilibrira in case of one-dimensional strategy spaces first for the case of two players and then for the case of $N$ players. Afterwards, I extend the results to the case of two-dimensional strategy spaces and $N \geq 2$. Then I introduce the condition for the inexistence of multiple symmetric equilibria. The section concludes with an application of these criteria to the Cournot game and a brief discussion of super- and submodular games.

\subsection{Review: criteria for uniqueness}

In order to determine whether or not a game has a unique equilibrium the literature has come up with three general approaches:

i) the contraction approach

ii) the univalence approach

iii) the Poincare-Hopf index theorem approach

I will first provide a short summary of the univalence approach and the index approach in the

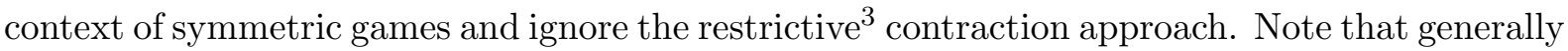

\footnotetext{
${ }^{3}$ It is straightforward to show that the contraction approach is a special case of the univalence approach (see Vives (1999)).
} 
nothing detains us from applying these methods to a symmetric game but these methods have in common that they rely on boundary conditions and usually are impractical in the context of particular applications as the matrices involved can be very large.

\subsubsection{The Univalence Approach}

The univalence approach developed by Gale and Nikaido (Gale and Nikaido (1965)) provides sufficient conditions under which a mapping is globally univalent (i.e. one-to-one). The following is a reformulation of the univalence theorem from Gale and Nikaido (p. 89) in the context of a symmetric game.

Theorem 1 (Gale-Nikaido) If all principal minors of $(-J(x))$ are positive for all $x \in S^{N}$ then at most one interior equilibrium can exist and this equilibrium must be symmetric.

The obvious shortcomings of the theorem are i) it says nothing about boundary equilibria, ii) it is impractical as determining the signs of all principal minors of a $N k \times N k$-matrix is very tedious and iii) it is quite easily violated in applications as the following example shows: ${ }^{4}$ Let $k=1$ and $N=2$ and suppose that $\Pi^{1}\left(x_{1}, x_{2}\right)=x_{1} \sqrt{x_{2}}-1 / 2 x_{1}^{2}$ and $\Pi^{2}\left(x_{1}, x_{2}\right)=x_{2} \sqrt{x_{1}}-1 / 2 x_{2}^{2}$. Then we have

$$
\nabla F(x)=\left(\begin{array}{c}
\sqrt{x_{2}}-x_{1} \\
\sqrt{x_{1}}-x_{2}
\end{array}\right) \quad S=[1 / 5,2]
$$

Then

$$
-J(x)=\left(\begin{array}{cc}
1 & -\frac{1}{2 \sqrt{x_{2}}} \\
-\frac{1}{2 \sqrt{x_{1}}} & 1
\end{array}\right)
$$

and $|-J(x)|=1-\frac{1}{4 \sqrt{x_{1} x_{2}}}$ which is not positive for all $\left(x_{1}, x_{2}\right) \in[1 / 5,2]^{2}$. Nevertheless, the symmetric equilibrium $(1,1)$ obviously is the only equilibrium.

\subsubsection{The index theorem approach}

The index theorem declares that, under assumption 2, we only need information about the sign of $\operatorname{Det}(-J(x))$ at critical points instead of investigating all principal minors of $-J(x)$. Suppose

\footnotetext{
${ }^{4}$ Although Rosen's version includes boundary equilibria (Rosen (1965)), the requirements of the payoff-function $\Pi$ are stronger (concavity), it involves checking the definitness of a large matrix and also fails to establish uniqueness in the example below.
} 
all equilibria points are regular and interior. The index of a zero of the vector field $\nabla F(x)$ is then defined by (see Vives (1999), p.48):

$$
I(x)= \begin{cases}+1 & \text { if } \operatorname{Det}(-J(x))>0 \\ -1 & \text { if } \operatorname{Det}(-J(x))<0\end{cases}
$$

It is known that if the strategy space forms a rectangle in some Euclidian space then $\mathcal{I} \equiv$ $\sum_{x \in E} I(x)=1$ where $I(x)$ is the index of a zero (see Simsek et al. (2007)). Hence if the boundary condition (assumption 2) holds and all equilibria points are regular then if

$$
\nabla F(x)=0 \quad \Rightarrow \quad \operatorname{Det}(-J(x))>0
$$

holds the symmetric equilibrium is unique. Note that in example (8) the index theorem obviously implies $(1,1)$ to be the unique equilibrium. ${ }^{5}$ The index approach may still be hard to verify in applications as the matrix involved can be very large. Moreover, in the case of boundary equilibria or non-regular equilibria ${ }^{6}$ the (standard) index theorem is not applicable ${ }^{7}$.

In the next two sections I develop the two criteria that together assert uniqueness of the symmetric equilibrium. I begin by discussing the possibility of multiple symmetric equilibria using the SOFA and then move on to the possibility of asymmetric equilibria.

\subsection{Multiplicity of symmetric equilibria}

In this section, based on the SOFA, I use the index theorem to establish a criterion that rules out the possibility of multiple symmetric equilibria. The set $E^{s}$ of interior symmetric equilibria is given by

$$
E^{s} \equiv\left\{\left(x_{1}, \ldots, x_{1}\right) \in S^{N}: \nabla \tilde{\Pi}\left(x_{1}\right)=0\right\}
$$

\footnotetext{
${ }^{5}$ The index approach is more general as univalence because condition (10) is implied if all principal minors of $-J(x)$ are found to be positive. Nevertheless, the Gale-Nikaido theorem is often invoked in applied work as it asserts the global invertibility of inverse demands (Vives (1999), p. 76).

${ }^{6}$ In such a case the zeroes of the gradient field are not locally isolated which violates a prerequisite of the index theorem (Vives, 1999, p. 362).

${ }^{7}$ Note that a generalised version of the index theorem has been proven (Simsek et al. (2007)), that can deal with boundary equilibria. However, this approach still requires to calculate the determinant of a large matrix, and further side conditions (non-degeneracy and complementarity) of what the authors term a generalised critical point must be verified separately.
} 
where $\nabla \tilde{\Pi}\left(x_{1}\right)$ is the gradient of $\tilde{\Pi}\left(x_{1}, \bar{x}\right)$ with respect to $x_{1}$, evaluated at $\bar{x}=x_{1}$ and

$$
\nabla \tilde{\Pi}\left(x_{1}\right): S \rightarrow \mathbb{R}^{k} \quad, x_{1} \mapsto \nabla \tilde{\Pi}\left(x_{1}\right)
$$

is a vector field over $S$. Let $\tilde{J}\left(x_{1}\right)$ denotes the Jacobian of $\nabla \tilde{\Pi}\left(x_{1}\right)$.

Assumption 3 (Symmetric boundary and regularity) Let $\nabla \tilde{\Pi}$ be the vector field as defined by (11). Then

i) $\nabla \tilde{\Pi}$ points inwards on the boundary of $S$

ii) $\operatorname{Det}\left(-\tilde{J}\left(x_{1}\right)\right) \neq 0$ if $\nabla \tilde{\Pi}\left(x_{1}\right)=0$

Proposition 3 Suppose assumption 3 is satisfied. Then all symmetric equilibria are interior and their number is odd. Further, if and only if

$$
\nabla \tilde{\Pi}\left(x_{1}\right)=0 \Rightarrow \operatorname{Det}\left(-\tilde{J}\left(x_{1}\right)\right)>0 \quad x_{1} \in \operatorname{Int}(S)
$$

then there is only one symmetric equilibrium.

Proof: Follows directly from the index theorem as $\nabla \tilde{\Pi}: S \rightarrow \mathbb{R}^{k}, x_{1} \mapsto \nabla \tilde{\Pi}\left(x_{1}\right)$ defines a vector field on $S$.

Let $\tilde{\Pi}_{i}\left(x_{1}\right)$ be the $i$-th component of $\nabla \tilde{\Pi}$. The boundary condition in proposition 3 then means that $\tilde{\Pi}_{i}\left(x_{11}, \ldots, 0_{i}, \ldots, x_{1 k}\right)>0$ and $\tilde{\Pi}_{i}\left(x_{11}, \ldots, \bar{S}_{i}, \ldots, x_{1 k}\right)<0$ for $1 \leq i \leq k$. Obviously, if assumption 2 is satisfied then also assumption 3 i) holds. But assumption 2 is unnecessarily restrictive $^{8}$ because we can restrict ourselves to $\nabla \tilde{\Pi}$ (rather than $\nabla \Pi^{1}$ ) which obviously reduces the complexity of the task. All points that violate the boundary condition in proposition 3 are candidates for symmetric boundary equilibria. If the boundary condition in proposition 3 fails to hold and it is known that interior symmetric equilibria exist we may apply the univalence approach to $\tilde{J}\left(x_{1}\right)$ : if all principal minors of $-\tilde{J}\left(x_{1}\right)$ are found to be positive for all $x_{1} \in S$ and an interior symmetric equilibrium exists, this is the only interior symmetric equilibrium of the game.

\footnotetext{
${ }^{8}$ If assumption 2 is satisfied the index theorem can be used to obtain a counting rule that reveals more regularities of the equilibrium set $E$ (see 6.1 in the appendix).
} 
As we will see in section 4.1 there is an intimate relationship between (symmetric) stability of symmetric equilibria and the multiplicity of such equilibria which makes the comparative statics problematic in case of multiple symmetric equilibria. Thus from this perspective it makes sense, as a modelling advice, to impose boundary conditions as stated by proposition 3 directly on the symmetric opponent form of the game.

\subsection{Inexistence of asymmetric equilibria}

In this section I develop a sufficient criterion that excludes asymmetric equilibria from the equilibrium set. I start with the simple case where $k=1$ and then generalise the result to the case $k=2$. Finally, I illustrate how to extend the criterion to $k \geq 3$.

\subsubsection{The case $k=1$}

Suppose $k=1$ and $N \geq 2$. Let $\varphi\left(x_{2} ; X\right) \equiv \varphi^{1}\left(x_{2} ; X\right)$ where $X \equiv\left(x_{3}, \ldots, x_{N}\right)$. Note that because of corollary 1 the partial derivative $\partial \varphi\left(x_{2} ; X\right) \equiv \frac{\partial}{\partial x_{2}} \varphi^{1}\left(x_{2} ; X\right)$ exists if $\varphi\left(x_{2} ; X\right) \in(0, \bar{S})$.

Theorem 2 If for all $x_{2} \in(0, \bar{S})$ and any given $X \in S^{N-2}$ for which $\partial \varphi\left(x_{2} ; X\right)$ exists we have that $\partial \varphi\left(x_{2} ; X\right)>-1$ then no asymmetric equilibrium exists.

Proof: Appendix (6.5)

Note that the shape of $\varphi\left(x_{2} ; X\right)$ is not restricted by theorem 2 up to the slope condition for those points $\left(x_{2} ; X\right)$ which imply that $\varphi\left(x_{2} ; X\right) \in(0, \bar{S})$. Hence, other than the index theorem, we do not require any boundary conditions to hold nor do we need to exclude the possibility of non-isolated asymmetric equilibria (i.e. theorem 2 does not require assumption 2). Moreover, to exclude asymmetric equilibria from the equilibrium set by theorem 2 we can focus on a two-player version of the game where the vector $X$ of strategies of the other player enter as exogenous parameters and the slope condition in theorem 2 is much simpler to work with than the determinant of a $N \times N$-matrix. A straightforward application of the IFT gives the following sufficient condition for the inexistence of asymmetric equilibria:

Corollary 2 If for all $x_{1}, x_{2} \in(0, \bar{S})$ and any given $X \in S^{N-2}$ we have that

$$
\Pi_{1}\left(x_{1}, x_{2} ; X\right)=0 \Rightarrow \frac{\Pi_{12}\left(x_{1}, x_{2} ; X\right)}{\Pi_{11}\left(x_{1}, x_{2} ; X\right)}<1
$$


then no asymmetric equilibrium exists.

Note that corollary 2 means that we can exclude asymmetric boundary equilibria by considering only interior points in a two-player game.

In the remainder of this section I provide the simple geometric intuition behind theorem 2 . In essence, it is an application of the Mean Value Theorem and the idea is illustrated in figure 1. Suppose the point $A=\left(x_{1}^{a}, x_{2}^{a}\right)$ corresponds to an asymmetric equilibrium of the game, as

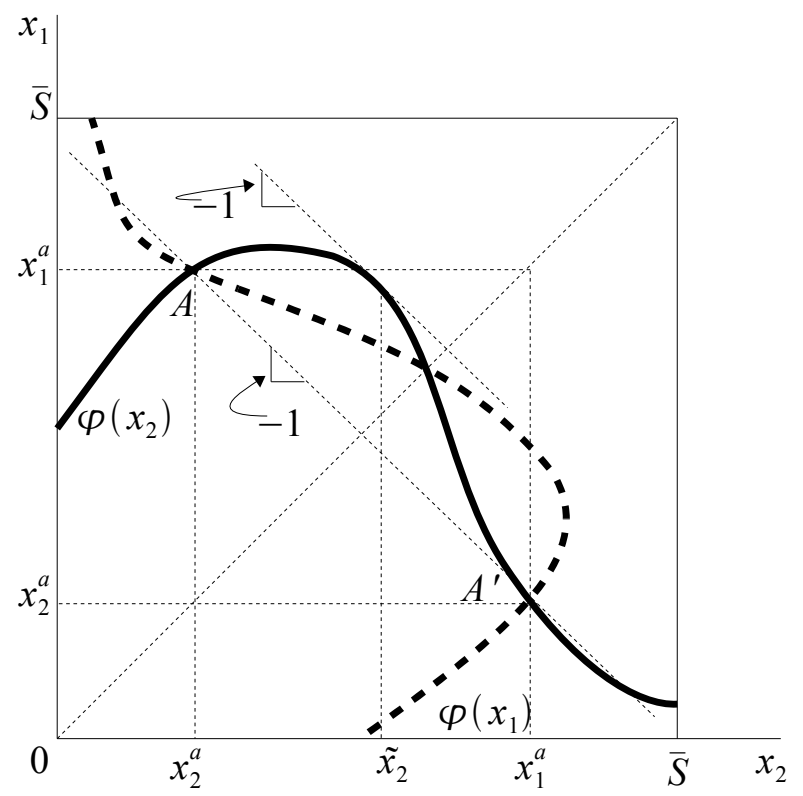

Figure 1: Theorem 2

depicted in the figure. By symmetry its reflection, the point $A^{\prime}=\left(x_{2}^{a}, x_{1}^{a}\right)$, must then also be an asymmetric equilibrium. As is depicted in the figure the line that connects $A$ and $A^{\prime}$ must have a slope of -1 . The best response function of player one, $\varphi\left(x_{2}\right)$, remains in the interior of $S=[0, \bar{S}]$ which according to corollary 1 means that this function must be differentiable for any $x_{2} \in(0, \bar{S})$. But then the Mean Value Theorem tells us that there must be a point $\tilde{x}_{2} \in\left(x_{2}^{a}, x_{1}^{a}\right)$ such that we have $\partial \varphi\left(\tilde{x}_{2}\right)=-1$. Theorem 2 then states that if we cannot find such a point $\left(\tilde{x}_{2}\right)$ we may conclude that no asymmetric equilibrium exists. ${ }^{9}$

\footnotetext{
${ }^{9}$ The complete proof (see appendix) is complicated by the fact that $\varphi\left(x_{2}\right)$ may be on the boundary and hence not differentiable everywhere.
} 


\subsubsection{The case $k=2$ and $N \geq 2$}

I now turn to the case where $k=2$. To ease notation I introduce the four symbols $\alpha, \beta, \gamma, \delta$ which denote the partial derivatives of the best response function of $g=1$ with respect to a strategy of player $g=2$ for given $\left(x_{21}, x_{22} ; X\right)$ where $X=\left(x_{31}, x_{32}, \ldots, x_{N 1}, x_{N 2}\right)$.

$$
\begin{aligned}
\alpha & \equiv \frac{\partial \varphi_{1}\left(x_{21}, x_{22} ; X\right)}{\partial x_{21}} \\
\beta & \equiv \frac{\partial \varphi_{1}\left(x_{21}, x_{22} ; X\right)}{\partial x_{22}} \\
\gamma & \equiv \frac{\partial \varphi_{2}\left(x_{21}, x_{22} ; X\right)}{\partial x_{21}} \\
\delta & \equiv \frac{\partial \varphi_{2}\left(x_{21}, x_{22} ; X\right)}{\partial x_{22}}
\end{aligned}
$$

Theorem 3 If for all $x_{2} \in \operatorname{Int}(S)$ and any given $X \in S^{N-2}$ for which the corresponding partial derivatives as defined by (14) exist we have that

$$
\begin{array}{cc}
\alpha>-1 \quad \delta>-1 \\
\alpha+\delta+\alpha \delta-\beta \gamma>-1
\end{array}
$$

then no asymmetric equilibrium exists.

Proof: Appendix (6.6)

Note that corollary 2 extends in a straightforward way to the case where $k=2$. Theorem 3 is not only a useful tool in order to exclude asymmetric equilibria (interior or boundary) from the equilibrium set but it also permits to get some economically interesting insights in case of two-dimensional games (see section 3.5.2). In the appendix (see 6.6) I illustrate that theorem 3 can be extended in a straightforward way to the case where $k>2$.

\subsection{Discussion}

If the conditions of proposition 3 and of theorem 2 or 3 (or the higher dimensional analogue) are satisfied, then the game only has one equilibrium, the interior symmetric equilibrium. Especially note that we only need to examine those boundary points that could be part of a symmetric boundary equilibrium and not the entire boundary - which is a clear practical advantage over the index theorem approach. Moreover, the slope condition of theorem 2 (or theorem 3) are simpler to evaluate than the index or the univalence condition as they depend only on the slope 
of the response function of an individual player with respect to a single other player and not on the Jacobian matrix of the entire game. Similarly, the condition of proposition 3 is simple to work with as it builds on the symmetric opponent form. Finally, as my approach to uniqueness finds two criteria which have a functional interpretation (one excludes asymmetric equilibria, one excludes multiple symmetric equilibria) we can also use these conditions to discuss economically interesting equilibrium features of a particular application.

\subsection{Applications and examples}

To demonstrate the generality of the separation approach develop so far I show in the next section that its application to the Cournot model generates two conditions asserting uniqueness which are weaker than the main conditions of uniqueness as worked up by the literature. The implications of theorem 3 for two-dimensional (supermodular) games are discussed afterwards.

\subsubsection{The Cournot game}

The Cournot model is one of the most analysed economic models. Let

$$
\Pi^{1}=q_{1} P(Q)-c\left(q_{1}\right) \quad Q=\sum_{i=1}^{N} q_{i}
$$

and $q_{i} \in[0, \bar{S}] \equiv S$. In accordance with assumption 1 I take $P(Q), c\left(q_{1}\right)$ to be twice continuously differentiable for $Q \in[0, N \bar{S}]$ and $q \in S$ with $P^{\prime}(Q)<0$ as well as $c^{\prime}\left(q_{1}\right)>0$ and $c^{\prime \prime}\left(q_{1}\right) \geq 0$ and assume strong quasiconcavity ${ }^{10}$. Then we have

$$
\nabla \tilde{\Pi}(q)=P(N q)+q P^{\prime}(N q)-c^{\prime}(q)
$$

The following assumption ${ }^{11}$ rules out symmetric boundary optima ${ }^{12}$

Assumption 4 (Cournot boundary conditions) The following symmetric boundary conditions are imposed:

${ }^{11}$ Note that this is the only boundary assumption required by the separation approach!

${ }^{12}$ If $\lim _{q \rightarrow 0} P(N q)+q P^{\prime}(N q)>0$ and $\lim _{q \rightarrow \infty} P(N q)+q P^{\prime}(N q) \leq 0$ hold and the function $\xi(q) \equiv P(N q)+q P^{\prime}(N q)$ is differentiable on $(0, \infty)$ we can always find $\underline{S}>0, \bar{S}<\infty$ such that $\nabla \tilde{\Pi}(\underline{S})>0$ and $\nabla \tilde{\Pi}(\underline{S})<0$ holds and hence proposition 3 is applicable (as taking zero to be the lower bound of the strategy space obviously can be relaxed in proposition 3).
} 
i) $\nabla \tilde{\Pi}(0)=P(0)-c^{\prime}(0)>0$

ii) $\nabla \tilde{\Pi}(N \bar{S})=P(N \bar{S})+\bar{S} P^{\prime}(N \bar{S})-c^{\prime}(\bar{S})<0$

Proposition 4 (Uniqueness in Cournot) Under assumption 4 and

i) if for $q \in(0, \bar{S})$ we have that

$$
q P^{\prime}(N q)+P(N q)-c^{\prime}(q)=0 \Rightarrow-\left(q N P^{\prime \prime}(N q)+P^{\prime}(N q)(1+N)-c^{\prime \prime}(q)\right)>0
$$

then only one symmetric equilibrium exists, and it is an interior equilibrium,

ii) if for $q_{1} \in(0, \bar{S})$ and any given $Q \in(0, N \bar{S})$ we have that

$$
P(Q)+q_{1} P^{\prime}(Q)-c^{\prime}\left(q_{1}\right)=0 \quad \Rightarrow \quad P^{\prime}(Q)-c^{\prime \prime}\left(q_{1}\right)<0
$$

then no asymmetric equilibrium exists.

iii) If (17) and (18) are both satisfied, then the Cournot game only has one equilibrium, the symmetric interior equilibrium

Proof: Appendix (6.7)

Note that by ii) we may restrict ourselves to interior points of $Q$ in order to exclude asymmetric (boundary) equilibria. Note that quasiconcavity does not imply that both conditions (17) and (18) need to hold but vice-versa if the two conditions hold then the payoff-function must be (srictly) quasiconcave at interior points.

How general are the conditions generated by the separation approach in case of the Cournot game? The condition of uniqueness ${ }^{13}$ as worked up by Gaudet and Salant are widely accepted as very general conditions of uniqueness (Gaudet and Salant (1991), p. 401). Usually, the requirements that

$$
\begin{aligned}
& \left(P^{\prime}+q_{1} P^{\prime \prime}\right)<0 \\
& c^{\prime \prime}\left(q_{1}\right)-P^{\prime}>0
\end{aligned}
$$

\footnotetext{
${ }^{13}$ If$$
\sum_{i \in M\left(Q^{E}\right)} \frac{P^{\prime}\left(Q^{E}\right)+q_{i}^{E} P^{\prime \prime}\left(Q^{E}\right)}{c^{\prime \prime}\left(q_{i}^{E}\right)-p^{\prime}\left(Q^{E}\right)}<1
$$

holds, where $M\left(Q^{E}\right)$ is the set of players for which a candidate equilibrium $q_{i}^{E}$ has $q_{i}^{\prime} E\left(Q^{E}\right)>0$, then only one equilibrium exists. Note that the Gaudet-Salant condition and the separation conditions depend on different (boundary) assumptions.
} 
hold is invoked in applied work (see e.g. Vives (1999), p. 98). It is not hard to see that these conditions imply the Gaudet-Salant condition as well as the separation conditions in proposition 4. Moreover, as $P^{\prime}(Q)-c^{\prime \prime}\left(q_{1}\right)<0$ is an exogenous assumption of Gaudet-Salant (which is not necessarily required by the assumptions of the separation approach!), we see that precisely this assumption excludes the possibility of asymmetric equilibria in the symmetric game (on and off the boundary). The separation conditions obviously are a lot simpler to evaluate than the Salant-Gaudet condition. Finally, we can give the two inequalities in (19) a new interpretation as they rule out different types of equilibria. Note that $P^{\prime \prime}(Q)$ does not occur in (18). This means that, under quasiconcavity, the curvature of $P(Q)$ plays no role for determining whether there are asymmetric equilibria or not. However, the curvature of $P(Q)$ (the elasticity of $P^{\prime}(Q)$ with respect to $q_{1}$ ) influences if there are multiple symmetric equilibria. In case of constant unit costs $\left(c^{\prime \prime}=0\right)$ it is easy to see that the possibility of asymmetric equilibria in the symmetric Cournot game is rather special: a necessary condition for the existence of asymmetric equilibria in this game is that $-P^{\prime} \leq 0$ is possible - which is contrary to the standard economic presumption of the model.

The fact that in the Cournot $N$-player game we may restrict ourselves to interior points of the aggregate quantity $Q$ is not a coincidence but stems from the fact that the strategies of the other players affect the payoff-function as a sum:

Corollary 3 If $\Pi\left(x_{1}, \ldots, x_{N}\right)=\hat{\Pi}\left(x_{1}, Q\right)$, where $Q \equiv \sum_{j=1}^{N} x_{j}$, and $\hat{\Pi}_{11}\left(x_{1}, Q\right)+\hat{\Pi}_{12}\left(x_{1}, Q\right)<0$ holds for any $x_{1} \in(0, \bar{S})$ and $Q \in(0, N \bar{S})$ with $\hat{\Pi}_{1}\left(x_{1}, Q\right)+\hat{\Pi}_{2}\left(x_{1}, Q\right)=0$, then no asymmetric equilibrium exists.

\section{Proof:}

In such a game we have $\Pi_{1}\left(x_{1}, \ldots, x_{N}\right)=\hat{\Pi}_{1}\left(x_{1}, Q\right)+\hat{\Pi}_{2}\left(x_{1}, Q\right), \Pi_{12}=\hat{\Pi}_{12}+\hat{\Pi}_{22}$ and $\Pi_{11}=$ $\hat{\Pi}_{11}+2 \hat{\Pi}_{12}+\hat{\Pi}_{22}$. Thus the claim follows directly from corollary 2 .

Note that the Cournot condition $\left(P^{\prime}-c^{\prime \prime}<0\right)$ is just a special case of corollary $3 .{ }^{14}$

\footnotetext{
${ }^{14}$ Corollary 3 does not generally extend to the case of a general aggregative game (see Alos-Ferrer and Ania (2005), p.500, for a definition of such games).
} 


\subsubsection{Super- and submodular games}

If $k=1$ and $N \geq 2$ a supermodular symmetric game has $\Pi_{12}\left(x_{j}, x_{-j}\right) \geq 0$ for all $x \in S^{N}$ whereas a submodular game has $\Pi_{12}\left(x_{j}, x_{-j}\right) \leq 0$. Thus with theorem 2 and proposition 3 we can easily replicate the result that supermodular games can never have any asymmetric equilibria ${ }^{15}$ but can have multiple symmetric equilibria whereas submodular games never have multiple symmetric equilibria but there can be asymmetric equilibria.

It is known that the inexistence result of asymmetric equilibria in supermodular games does not extend to the case of multi-dimensional strategy spaces. That two-dimensional symmetric supermodular games may have asymmetric equilibria has been shown to hold by means of (nondifferentiable) examples (e.g. Amir et al. (2008), p. 311). Let $N=2$. From (15) we see that a necessary condition for asymmetric equilibria to occur is that $\beta=\frac{\partial \varphi_{1}\left(x_{21}, x_{22}\right)}{\partial x_{22}}$ and $\gamma=\frac{\partial \varphi_{2}\left(x_{21}, x_{22}\right)}{\partial x_{21}}$ are large compared to $\alpha=\frac{\partial \varphi_{1}\left(x_{21}, x_{22}\right)}{\partial x_{21}}$ and $\delta=\frac{\partial \varphi_{1}\left(x_{21}, x_{22}\right)}{\partial x_{21}}$. This result is intuitive as $\beta$ and $\gamma$ refer to cross-over effects (e.g. how does a change of advertising intensity of my competitor affect my pricing decision) of the strategies whereas $\alpha$ and $\delta$ capture the direct effects (e.g. how does a price change of my competitor affect my pricing decision). Hence weak direct complementarity but strong cross-over complementarity is necessarily required to generate asymmetric equilibria. Suppose that two players play a supermodular price-advertising game as indicated above with the property that there is a very strong cross-over but a weak direct complementarity. Now assume that player two increases his price. This induces player one also to increase his price and advertising but, because of the strong cross-over complementarity, he increases advertising by more. This in turn means that player two continues to increase his price. Hence this type of "dynamic" reinforcement intuitively explains the economically interesting result why asymmetric "specialisation" equilibria in symmetric two-dimensional supermodular games may in fact occur. However, if the game is supermodular $(k>1)$ and proposition 3 holds (such that there only is one symmetric equilibrium) then no asymmetric equilibrium can exist. ${ }^{16}$ Hence for supermodular games proposition 3 is necessary and sufficient for uniqueness of the (symmetric interior) equilibrium.

In the case of a submodular game an evaluation of (12) shows that already for $k=2$ such a

\footnotetext{
${ }^{15}$ Note that example (8) is a supermodular game. Hence the failure of the univalence approach in this example is only driven by the fact that there could be multiple symmetric equilibria.

${ }^{16}$ This is a consequence of the fact that in supermodular symmetric games the extremal equilibria must always be symmetric; Vives (2005), p. 448.
} 
game can possibly generate multiple symmetric equilibria.

Many economically interesting applications involve the case of two-dimensional strategy spaces. We can extract some modelling advice from theorem 3 to exclude asymmetric equilibria from the equilibrium set. Suppose that $\alpha, \delta>-1$ is known to hold. Especially, games were the strategies are nested in the sense that $\beta, \gamma \neq 0$ are interesting as such games cannot be solved independently for each strategy. The following properties then assert inexistence of asymmetric equilibria (on and off the boundary) of such games:

- Either $\beta$ or $\gamma$ is zero at least at equilibrium candidates. ${ }^{17}$

- $\alpha, \delta>0$ and $|\beta|,|\gamma| \leq 1$.

- $\beta \geq 0$ and $\gamma \leq 0$ (or vice-versa).

Finally, it is not hard to see by using the implicit function theorem that if

$$
\begin{aligned}
& \Pi_{1}\left(x_{11}, x_{12}, x_{21}, x_{22} ; X\right)=0 \\
& \Pi_{2}\left(x_{11}, x_{12}, x_{21}, x_{22} ; X\right)=0
\end{aligned} \quad \Rightarrow \quad\left|\Pi_{i i}\right|>\sum_{j \neq i, j \leq 4}\left|\Pi_{i j}\right| \quad i=1,2
$$

holds for any $\left(x_{1}, x_{2}\right) \in \operatorname{Int}\left(S^{2}\right)$ and any given $X \in S^{N-2}$ then $\alpha, \delta>-1$ as well as $1+\alpha, 1+\delta>$ $\beta, \gamma$ and the game cannot have any asymmetric equilibria ${ }^{18}$.

\subsubsection{Monotonic one-dimensional games}

In this section I illustrate how the investigation of the equilibrium set of a symmetric game can provide important insights into the equilibrium set of certain asymmetric variations of the game. For simplicity, I concentrate on the case where the heterogeneity of the payoff-functions is restricted to the distribution of one parameter, e.g. the Cournot-competitors have cost functions $c_{1} q_{1}, \ldots, c_{N} q_{N}$. Suppose $k=1$ and let the payoff-functions be $\Pi\left(x_{g}, x_{-g} ; c_{g}\right)$, where $c_{g} \in[\underline{\mathrm{c}}, \bar{c}]$ is a parameter. Thus if $c_{g} \neq c_{h}$ for some $g \neq h$ we have an asymmetric game whereas if $c_{g}=c_{h}=c$ for all players the game is symmetric.

\footnotetext{
${ }^{17}$ E.g. if the best-response subfunction $\varphi_{1}\left(x_{2}, \ldots, x_{N}\right)$ depends only on the sub-strategy vector $\left(x_{j 1}\right)_{2 \leq j \leq N}$ then no asymmetric equilibrium can exist - independent of how $\varphi_{2}$ depends on $x_{-1}$.

${ }^{18}$ Hence if the Jacobian matrix $J(x)$ of $\nabla F(x)$ as defined by (5) has a dominant diagonal then condition (14) is trivially implied.
} 
The symmetric game $\left(N, S^{N},\left\{\Pi^{g}(x ; c)\right\}_{g=1}^{N}\right)$ is (strictly) monotonic on $[\underline{\mathrm{c}}, \bar{c}]$ if the reaction function of each player is (strictly) monotonic in the parameter $c$ :

$$
c^{\prime}>c \Rightarrow \varphi\left(x_{-g}, c^{\prime}\right) \stackrel{(>)}{\geq} \varphi\left(x_{-g}, c\right)
$$

The following proposition reveals a strong relationship between the (in)existence of asymmetric equilibria in symmetric games and the ranking of the strategies in any equilibrium of an asymmetric version of the game. To be precise I consider the case where $\bar{c} \geq c_{1}>c_{2}, \ldots,>c_{N}=\underline{\mathrm{c}}$. Then $\left(N, S^{N},\left\{\Pi^{g}\left(x ; c_{g}\right)\right\}_{g=1}^{N}\right)$ is an asymmetric game and any future reference to the asymmetric game refers to this game. Let the binary symmetric game be defined as the game where players $g$ and $g+1$ play a symmetric game $\left(c_{g}=c_{g+1}\right)$ where $X_{g} \equiv x \backslash\left\{x_{g}, x_{g+1}\right\} \in S^{N-2}$ is an exogenous vector of parameters.

Proposition 5 Suppose $\left(N, S^{N},\left\{\Pi^{g}(x ; c)\right\}_{g=1}^{N}\right)$ is monotonic on $[\underline{c}, \bar{c}]$

i) If for $c=\underline{c}$ the binary symmetric game has no asymmetric equilibrium for any given $X_{g} \in S^{N-2}$ then any equilibrium $\left(x_{1}^{*}, \ldots, x_{N}^{*}\right)$ of the asymmetric game has the property that $x_{1}^{*} \geq x_{2}^{*} \geq, \ldots, \geq x_{N}^{*}$.

ii) If for any $c \in[\underline{c}, \bar{c}]$ the binary symmetric game has no asymmetric equilibrium for any given $X_{g} \in S^{N-2}$ and the game is strictly monotonic on $[\underline{c}, \bar{c}]$ then $x_{1}^{*}>x_{2}^{*}>\ldots>x_{N}^{*}$.

iii) If for any given $c \in[\underline{c}, \bar{c}]$ the interior symmetric equilibrium $x^{s}(c)$ is the unique equilibrium and the game is strictly monotonic on $[\underline{c}, \bar{c}]$ then $x_{1}^{*}>x_{2}^{*}>\ldots>x_{N-1}^{*}>x^{s}(\underline{c})$.

Proof: Appendix (6.8)

Whether or not we even have $x_{N}^{*}>x^{s}(\underline{\mathrm{c}})$ in iii) additionaly depends on the complementarity in the (symmetric) game. If the strategies are substitutes, i.e. the reaction functions are strictly decreasing, then we must have $x_{N}^{*}<x^{s}(\underline{\mathrm{c}})$ whereas under strict complements the opposite holds. Note that the requirement of the binary symmetric game not to have asymmetric equilibria is precisely the condition of theorem 2 asserting that the symmetric $N$-player game has no asymmetric equilibrium. 
Finally note that under differentiability the symmetric game obviously is monotonic on $[\underline{c}, \bar{c}]$ if and only if

$$
\frac{\partial \Pi\left(x_{g}, x_{-g} ; c\right)}{\partial x_{g}}=0 \Rightarrow \frac{\partial^{2} \Pi\left(x_{g}, x_{-g} ; c\right)}{\partial x_{g} \partial c} \geq 0 \quad x_{-g} \in S^{N-1}, c \in[\underline{c}, \bar{c}]
$$

\section{Stability}

In this section I discuss the connection between (local) stability and symmetric equilibria. For example, Dastidar shows that in case of the symmetric Cournot game uniqueness of the equilibrium and its local stability are intimately related (Dastidar (2000), p.213). By introducing the concept of symmetric stability I show for one-dimensional games satisfying assumption 3 that local symmetric stability and the inexistence of multiple symmetric equilibria are in fact the same properties. However, for higher dimensional games stability is a stronger requirement than non-multiplicity of symmetric equilibria. Finally, by exploiting the SOFA, I derive a condition that asserts whether an interior symmetric equilibrium of a $k$-dimensional game is a local contraction which is more general than the usual argument of local diagonal dominance.

\subsection{Comparative statics and symmetric stability}

As we consider static games, stability conditions are in any case "without foundation" (Dixit (1986), p. 107). Nevertheless, as Dixit highlights, there is a close link between the sign of a comparative-static prediction and the local stability of an equilibrium. It turns out that a modification of Dixit's concept of local myopic stability provides a fundamental link between stability under symmetric deviations and the (in)existence of multiple symmetric equilibria.

To illustrate the importance of such a consideration I reexamine the Cournot example from section 3.4.1. I assume that assumption 3 is satisfied and work with linear costs for simplicity: $c\left(q_{1}\right)=c q_{1}$. Let $q \in(0, \bar{S})$ denote a symmetric interior equilibrium. Then by the implicit function theorem:

$$
q^{\prime}(c)=\frac{1}{N P^{\prime \prime}(Q) q+(1+N) P^{\prime}(Q)}
$$

We immediately recognize $N P^{\prime \prime}(Q) q+(1+N) P^{\prime}(Q)$ as the relevant term in (17) that determines whether or not there are multiple symmetric equilibria. Thus in the case of multiple symmetric 
equilibria we can get $^{19} q^{\prime}(c)>0$ at a particular equilibrium point. In such a case we must have that strategic effects are of first-order importance, i.e. they reverse the direction of the comparative-statics as suggested by the direct effect. To see this consider an exogenous, symmetric change of unit $\operatorname{costs} c$. Assuming that all competitors choose the same initial response $d \bar{q}$, the change of strategy of firm one, $d q_{1}$, can be determined by using the total differential of the first-order condition of the symmetric opponents form:

$$
\underbrace{(N-1)\left(P^{\prime \prime}(Q) q_{1}+P^{\prime}(Q)\right)}_{A} d \bar{q}+\underbrace{\left(P^{\prime \prime}(Q) q_{1}+2 P^{\prime}(Q)\right)}_{B} d q_{1}-d c=0
$$

From section 3.4.1 we know that $P^{\prime \prime}(Q) q_{1}+P^{\prime}(Q)>0$ is necessary for multiple interior symmetric equilibria to exist and $B<0$ follows from strong quasiconcavity. The direct effect of the $c$-shock on $q_{1}, \frac{d q_{1}^{D}}{d c}$ can be found by holding the competitors' reaction fixed, i.e. $d \bar{q}=0$. Then $\frac{d q_{1}^{D}}{d c}=1 / B$ and thus $\frac{d q_{1}^{D}}{d c}<0$. The strategic effect is $\frac{d q_{1}}{d \bar{q}}=-A / B$ and corresponds to the slope of the bestresponse function $q_{1}(\bar{q})$ as defined by the FOC of the symmetric opponent form. From (21) we get that $\frac{d q_{1}}{d c}=\frac{1}{B}-\frac{A}{B} \frac{d \bar{q}}{d c}$. Hence in equilibrium $\left(d q_{1}=d \bar{q}=d q\right)$ :

$$
\frac{d q}{d c}=\frac{1}{B} \frac{B}{A+B}=\frac{1}{A+B}
$$

Thus we have $\operatorname{sign}\left(\frac{d q}{d c}\right)=\operatorname{sign}\left(\frac{d q_{1}^{D}}{d c}\right)$ if and only if $-B>A$ which is equivalent to the slope of $q_{1}(\bar{q})$ being less than one at an equilibrium point. Thus if only one symmetric equilibrium exists then we must have that $\operatorname{sign}\left(\frac{d q}{d c}\right)=\operatorname{sign}\left(\frac{d q_{1}^{D}}{d c}\right)$, i.e. strategic effects are of second-order importance (they influence only the magnitude of the change). Note that this condition is independent of whether there are asymmetric equilibria or not. Although (local) stability under symmetric adjustments is a restricted version of general myopic stability ${ }^{20}$ this analysis uncovers that symmetric stability is a minimal consistency requirement for the comparative statics of a game and the inexistence of multiple symmetric equilibria asserts symmetric stability in the Cournot example.

I now establish the link between symmetric stability and multiplicity of symmetric equilibria more formally.

\footnotetext{
${ }^{19}$ Because proposition 3 is an index theorem result, in the case of multiple symmetric regular equilibria we must have $N P^{\prime \prime}(Q) q+(1+N) P^{\prime}(Q)>0$ for at least one equilibrium point $q$

${ }^{20} \mathrm{~A}$ symmetric equilibrium that is stable under symmetric adjustments need not be stable under general myopic adjustments.
} 
Following Dixit's approach to stability I define the myopic adjustment process by

$$
\begin{aligned}
& \dot{x}_{11}=s_{1} \frac{\partial}{\partial x_{11}} \Pi\left(x_{1}, x_{-1}\right) \\
& \vdots \\
& \dot{x}_{1 k}=s_{k} \frac{\partial}{\partial x_{1 k}} \Pi\left(x_{1}, x_{-1}\right) \\
& \vdots \\
& \dot{x}_{N k}=s_{k} \frac{\partial}{\partial x_{N k}} \Pi\left(x_{N}, x_{-N}\right)
\end{aligned}
$$

where $s_{1}, \ldots, s_{k}>0$ are arbitrary adjustment speeds (Dixit (1986)). A solution to (22) has the form $x(t)=\left(x_{j}(t)\right)_{1 \leq j \leq N}$, where $x_{j}(t)=\left(x_{j 1}(t), \ldots, x_{j k}(t)\right)$ is the vector trajectory of player $j$. The symmetric myopic adjustment process is a restricted version where we require the initial values $x(0)$ to be symmetric, i.e. $x_{j}(0)=x_{i}(0), 1 \leq i, j \leq N$. Under this restriction the time path $x_{j}(t)$ must be the same for all players and is the solution to

$$
\begin{aligned}
& \dot{x}_{11}=s_{1} \tilde{\Pi}_{1}\left(x_{1}\right) \\
& \vdots \\
& \dot{x}_{1 k}=s_{k} \tilde{\Pi}_{k}\left(x_{1}\right)
\end{aligned}
$$

Let $\hat{J}\left(x_{1}\right)$ denote the Jacobian corresponding to $(23)$ and suppose that $x=\left(x_{1}, \ldots, x_{1}\right)$ is an interior symetric equilibrium. The point $x$ is symmetrically stable (i.e. stable under symmetric adjustments) if $\hat{J}\left(x_{1}\right)$ only has negative eigenvalues (or eigenvalues with negative real part).

Proposition 6 Suppose that $x^{*} \in E^{s}$ is locally symmetrically stable. Then there exists $\varepsilon>0$ such that for any initial value $x(0)$ on the $k$-dimensional symmetric manifold

$$
M\left(x^{*}\right) \equiv\left\{x \in S^{N}: x_{i}=x_{j} \forall 1 \leq i, j \leq N\right\} \cap \mathbb{B}\left(x^{*}, \varepsilon\right)
$$

the system (22) has a uniques solution with $x(t) \rightarrow x^{*}$.

Proof: Appendix (6.9)

Figure 2 illustrates the relastionship between stability and symmetric stability in the simplest possible case $((k, N)=(1,2))$.

If $\lambda=\left(\lambda_{1}, \ldots, \lambda_{k}\right)$ are the eigenvalues of $\hat{J}\left(x_{1}^{*}\right)$, then $\lambda$ must also be eigenvalues of $\hat{J}\left(x^{*}\right)$, the 

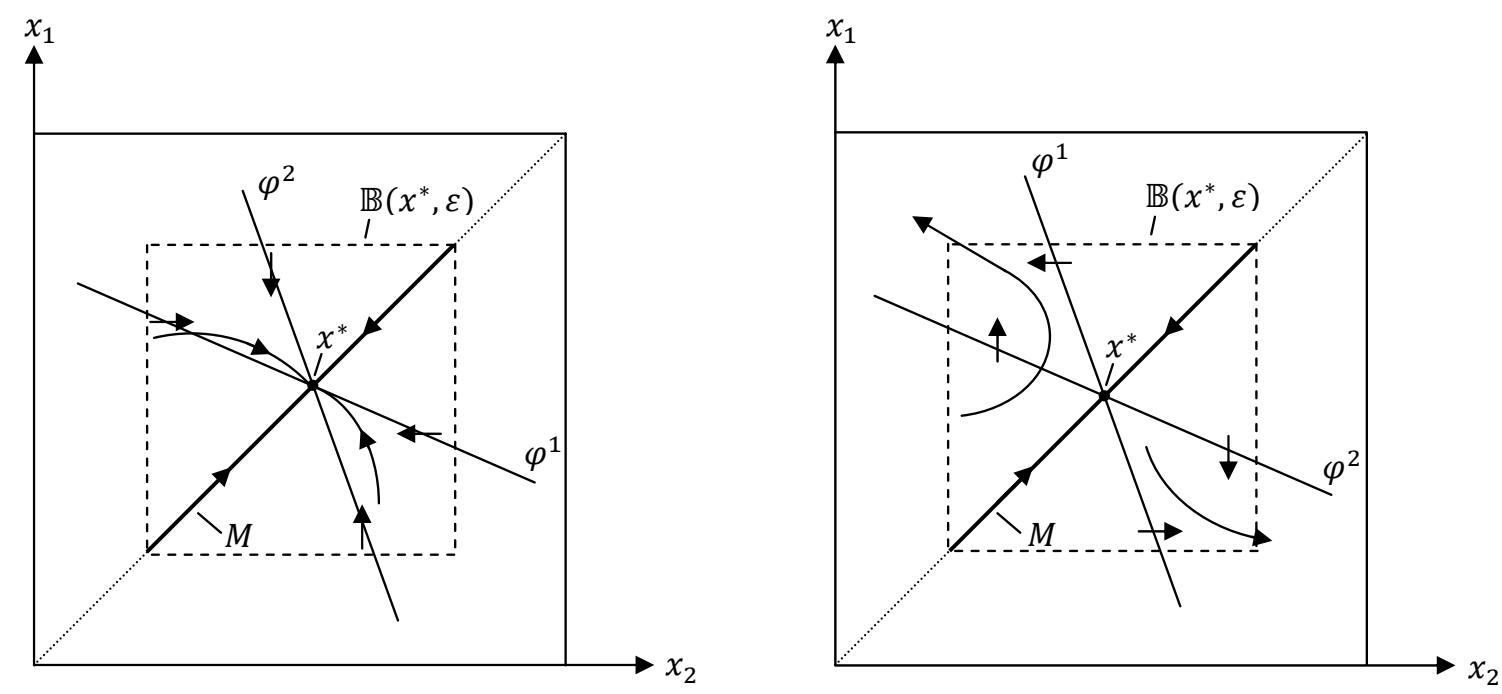

Figure 2: Generally stable eq. (left) and only symmetrically stable eq.

Jacobian corresponding to (22) (see appendix 6.9). Consequently, if $\lambda$ consists only of eigenvalues with negative real part then $\hat{J}\left(x^{*}\right)$ must have at least $k$ eigenvalues with negative real part and, by the saddle path theorem, there exists a $k$-dimensional manifold about $x^{*}$ on which $x(t)$ converges to $x^{*}$. Proposition 6 shows that this manifold simply takes on the form of a symmetric (topological) subspace about the equilibrium $x^{*}$. This makes clear why the requirement of symmetric stability, rather than the stronger requirement of general myopic stability, is reasonable if the (symmetric) comparative-statics of the symmetric equilibrium are to be investigated. Suppose we initially are at a symmetric equilibrium $x^{*}$ and that a set of parameters of the game change identically for all players (e.g. all players get access to the same cheaper production technology). Then the reaction functions shift according to this change and, assuming that a new symmetric equilibrium $x^{* *}$ exists, the former equilibrium is an initial value for the dynamic system (22) of the new equilibrium at the moment of the change: $x(0)=x^{*}$. If the change is sufficiently small (such that $x^{*} \in M\left(x^{* *}\right)$ ) then, by proposition 6 , we have that $x(t) \rightarrow x^{* *}$.

The following proposition reveals the relationship between symmetric stability and (non)-multiplicity of symmetric equilibria.

Proposition 7 (Symmetric stability and uniqueness) Suppose that assumption 3 is satisfied. Then the following statements hold:

i) If for $k \geq 1$ multiple symmetric equilibria exist, then there must be (symmetrically) unstable 
symmetric equilibria.

ii) If for $k=2$ we have that

$$
\nabla \tilde{\Pi}\left(x_{1}\right)=0 \quad \Rightarrow \quad \tilde{\Pi}_{11}\left(x_{1}\right), \tilde{\Pi}_{22}\left(x_{1}\right)<0, \operatorname{Det}\left(\tilde{J}\left(x_{1}\right)\right)>0
$$

then there only is one symmetric equilibrium and it is locally symmetrically stable.

iii) For $k=1$ the following statements are equivalent:

(a) All symmetric equilibria are locally symmetrically stable

(b) There only exists one symmetric equilibrium

(c) Strategic effects are of second-order importance at symmetric equilibrium points:

$$
\operatorname{sign}\left(x_{1}^{\prime}(c)\right)=\operatorname{sign}\left(\frac{\partial}{\partial c} \tilde{\Pi}_{1}\left(x_{1}, c\right)\right)
$$

Moreover, if $x$ is a symmetrically stable equilibrium with $\Pi_{11}(x)<\Pi_{12}(x)$ then $x$ is a stable equilibrium.

Proof: Appendix (6.10)

An immediate consequence of i) is that at any symmetrically stable equilibrium we must have $\operatorname{Det}\left(-\tilde{J}\left(x_{1} *\right)\right)>0$ (i.e. $x_{1}^{*}$ has a positive index). Further, we see that symmetric stability and non-multiplicity of symmetric equilibria are the same properties of one-dimensional games. In such games, instability of an equilibrium can be caused only by dominant aggregate strategic effects. This close connection between non-multiplicity and stability generally breaks down in

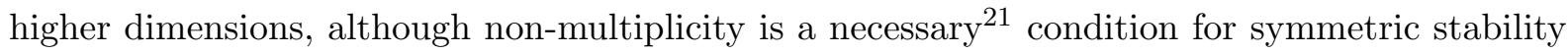
(and hence also for general myopic stability). Hence stability is a stronger concept than uniqueness (see proposition 8 below) and not much can be said in general concerning the relationship between stability and the sign of the comparative statics. However, as argued above the requirement of a symmetric interior equilibrium $x^{*}$ to be at least stable under symmetric adjustments is a reasonable prerequirement for comparative statics and also puts

The following condition is sufficient for symmetric stability and uniqueness ${ }^{22}$ for any $k \geq 1$ :

\footnotetext{
${ }^{21}$ It should be noted that for $k>1$ non-multiplicity of symmetric equilibria generally is not sufficient for local symmetric stability.

${ }^{22}$ In the literature many different sufficient conditions for stability have been proposed. See Tang et al. (2007) for a modern survey on matrix stability.
} 
Proposition 8 (Sufficient condition for symmetric stability) If the symmetric matrix $\hat{M}\left(x_{1}\right) \equiv-\left(\hat{J}\left(x_{1}\right)+\hat{J}\left(x_{1}\right)^{T}\right)$ is positive definite for any choice of $s_{1}, \ldots, s_{k}>0$ then $x=$ $\left(x_{1}, \ldots, x_{1}\right)$ is a locally symmetrically stable equilibrium. Further, if

$$
\nabla \tilde{\Pi}\left(x_{1}\right)=0 \quad \Rightarrow \quad \hat{M}\left(x_{1}\right) \text { positive definite }
$$

then there only is one symmetric equilibrium and it is locally symmetrically stable.

Proof: Appendix 6.11

In the case of supermodular games the sufficient condition for stability becomes particularly simple:

Proposition 9 (Symmetric stability in supermodular games) If the game is supermodular and for $\nabla \tilde{\Pi}\left(x_{1}\right)=0$ we have that all principal minors of $-\tilde{J}\left(x_{1}\right)$ are positive then $x=$ $\left(x_{1}, \ldots, x_{1}\right)$ is a locally symmetrically stable equilibrium point.

Proof: Appendix 6.12

\subsection{Local contraction stability}

In the literature on stability also discrete (or iterative) adjustment processes have been considered. I present a condition for an equilibrium to be a local contraction that depends only on the symmetric opponent form which should provide useful in applications and is more general than the requirement of diagonal dominance if $k>1$.

Let $\tilde{\varphi}_{i}(\bar{x})$ denote the $i$-th component of the $k$-vector $\tilde{\varphi}(\bar{x})$ as introduced in section 2.1 and $\bar{x}=\left(\bar{x}_{1}, \ldots, \bar{x}_{k}\right) \in S$.

Theorem 4 Suppose $x^{*} \in E^{s}$. If

$$
\max _{1 \leq i \leq k}\left\{\sum_{j=1}^{k}\left|\frac{\partial \tilde{\varphi}_{i}\left(\bar{x}^{*}\right)}{\partial \bar{x}_{j}}\right|\right\}<1
$$

then there exists $\varepsilon>0$ such that $\phi(x)$ is a contraction on $V \equiv \overline{\mathbb{B}}(x, \varepsilon)$. 
Proof: Appendix (6.13)

In words, condition (25) means that, for any subfunction $i$, the sum of the absolute values of the partial derivatives ${ }^{23}$ with respect to the symmetric opponent $k$-vector $\bar{x}$ must be less than one. It should be noted that this approach to stability puts no restriction on the initial deviations of the players other than they must be in the contraction basin $V$ (i.e. close to the equilibrium). In particular, initial deviations need not be symmetric. This is somewhat remarkable as condition (25) only makes use of the symmetric opponent form, i.e. we do not have to work with the entire payoff-function which considerably simplifies the analysis for higher dimensional games or games with complicated payoff-functions. Moreover, by using the IFT, condition (25) should be simpler to evaluate in higher-dimensional applications than the corresponding myopic stability condition on the eigenvalues of $J(x)$. For example, in two-dimensional games we only need to calculate the four partial derivatives corresponding to the symmetric opponent form (irrespective of the number of players!) whereas myopic stability requires to examine the eigenvalues of a (generally non-symmetric) $2 N \times 2 N$-matrix.

The usual sufficient condition for $\phi$ to be a contraction is that the Jacobian $J(x)$ has a dominant diagonal (e.g. Vives (1999), 1999, p. 47). For $k=1$ diagonal dominance and (25) are the same requirement. If $k>1$ then it is not hard to find an example (see 6.13 in the appendix) that shows condition (25) to be more general than the requirement of a dominant diagonal.

\section{Conclusion}

In this paper I developed a comparably simple approach to uniqueness by deriving two criteria which together assert uniqueness of the interior symmetric equilibrium of a differentiable symmetric game with compact strategy space. One criterion excludes the possibility of multiple symmetric equilibria and one criterion excludes the possibility of asymmetric equilibria. I demonstrated the generality of my approach by applying it to the Cournot game. Further, I have used the criterion that deals with asymmetric equilibria to learn more about what general properties of symmetric two-dimensional games can exclude asymmetric equilibria (on or off the boundary) from the equilibrium set. An application of my approach to asymmetric games revelas that certain equilibrium properties of asymmetric games may be found by investigat-

\footnotetext{
${ }^{23}$ In applications, these derivatives can be calculated directly from the equilibrium condition $\nabla \tilde{\Pi}\left(x_{1}, \bar{x}\right)=0$ by a direct application of the Implicit Function Theorem.
} 
ing the underlying symmetric game. Finally, the connection between multiplicity of symmetric equilibria and symmetric stability has been discussed and a condition for an interior symmetric equilibrium to be a local contraction was developed that is more general than the standard requirement of a dominant diagonal.

\section{Appendix}

\subsection{A counting rule}

Suppose assumption 2 is satisfied (so that only interior equilibria exist) and all equilibrium points are regular. If $x^{a}$ is an asymmetric equilibrium then by the permutation-invariance property of $\nabla F$ for every possible alignment $\tilde{x}^{a}$ of the vectors $\left(x_{1}^{a}, \ldots, x_{N}^{a}\right)$ of $x^{a}$ we must have that $\tilde{x}^{a}$ also is an asymmetric equilibrium. Thus it makes sense to introduce the notion of a distinct asymmetric equilibrium. Let $\left(x_{1}, x_{2}, \ldots, x_{N}\right),\left(x_{1}{ }^{\prime}, x_{2}{ }^{\prime}, \ldots, x_{N}^{\prime}\right) \in S^{N}$ denote two asymmetric equilibria. Then these are distinct asymmetric equilibria if they are not permutations of each other.

Proposition 10 Suppose assumption 2 is satisfied and all equilibria points are regular. Let $\mathcal{I}^{s}$ denote the sum of the indices of all symmetric equilibria on the field of $\nabla F$ defined in (7).

1) If $\mathcal{I}^{s}=1$, then if there are asymmetric equilibria

i) if $N \geq 2$ there must be more than one distinct asymmetric equilibrium

ii) if $N=2$ there must be an even number of distinct asymmetric equilibria

2) If $\mathcal{I}^{s} \neq 1$ and $N \geq 2$ then asymmetric equilibria exist. If especially $N=2$ then

i) if $\mathcal{I}^{s}=3+4 z$ for $z \in \mathbb{Z}$ then there must be an odd number of distinct asymmetric equilibria

ii) if $\mathcal{I}^{s}=5+4 z$ for $z \in \mathbb{Z} \backslash\{-1\}$ then there must be an even number of distinct asymmetric equilibria

Proof:

Because all equilibrium points are regular every equilibrium point has a well defined index which is either +1 or -1 (see (9)). Let $\omega \geq 1$ be the number of symmetric equilibria which is odd by proposition 3. Then $\mathcal{I}^{s}$ must be a number from $\{ \pm 1, \pm 3, \pm 5, \ldots, \pm \omega\}$. Let $\mathcal{I}^{a}$ denote the index 
sum of all asymmetric equilibria. Then we must have $\mathcal{I}^{s}+\mathcal{I}^{a}=1$ because $S^{N}$ is a rectangle ${ }^{24}$. If $\mathcal{I}^{s}=1$ but there are asymmetric equilibria then $\mathcal{I}^{a}=0$ which requires at least two distinct asymmetric equilibria because all non-distinct asymmetric equilibria must have the same index. This proves 1$)$ i). If $\mathcal{I}^{s} \neq 1$ we must have $\mathcal{I}^{s} \neq 0$ which proves that asymmetric equilibria must exist.

To see the rest set $N=2$ and note that with $N=2$ non-distinct asymmetric equilibria can only appear pairwise. Let $n_{1}$ denote the number of distinct asymmetric equilibria with index -1 and $n_{2}$ those with index +1 . Then the index theorem implies that $n_{2}-n_{1}=\frac{1-\mathcal{I}^{s}}{2}$. Now, if $\mathcal{I}^{s}$ is a number $3+4 z$ the RHS of this equation is an odd number which means that either $n_{1}$ or $n_{2}$ must be odd and the other number must be even or zero. Consequently, $n_{1}+n_{2}$ must be odd. For $\mathcal{I}^{s}=5+4 z$ with $z \in \mathbb{Z} \backslash\{-1\}$ the RHS must be even and hence $n_{1}+n_{2}$ must be even. Finally, if $\mathcal{I}^{s}=1$ we must have $n_{2}=n_{1}=n$. If $n>0$ then this implies $n_{1}+n_{2}=2 n$ which always is even.

\subsection{Proof of proposition 1}

Let $\varphi\left(x_{-g}\right)$ denote the set of maximisers of player $g$ from problem (2). Then $\varphi\left(x_{-g}\right)$ is nonempty by the continuity of $\Pi$ and the compactness of $S$. Moreover, $\varphi\left(x_{-g}\right)$ is single-valued for each feasible $x_{-g}$ because of the strict quasiconcavity of $\Pi$ in $x_{g}$ and the convexity of $S$. Finally, the continuity of $\Pi$ implies the continuity of $\varphi\left(x_{-g}\right)$ which, by symmetry, implies the continuity of $\phi$. For the second claim note that $\tilde{\Pi}\left(x_{1}, \bar{x}\right)$ is continuous and strongly quasiconcave in $x_{1}$ by the properties of $\Pi^{1}$. Hence the best-response function $\tilde{\varphi}(\bar{x}) \equiv \varphi\left(\bar{x}_{-1}\right)$ exists, is continuous and a mapping from $S$ to itself. The result then follows from the Brouwer FPT.

\subsection{Proof of proposition 2}

First note that because $\Pi^{g}$ is $C^{2}$ for $g=1, \ldots, N$ the gradient field $\nabla F$ is well-defined. Because of symmetry we can concentrate on player $g=1$ without loss of generality. Let $x=\left(x_{1}, x_{-1}\right)$ and suppose $x_{1} \in \partial S$, i.e. for at least one $x_{1 i}$ with $1 \leq i \leq k$ we have that $x_{1 i} \in\left\{0, \bar{S}_{i}\right\}$. If $x_{1 i}=0$

\footnotetext{
${ }^{24} \mathrm{~A}$ rectangle has an Euler characteristic of one which must correspond to the index sum of the vector field on the rectangle (see Simsek et al. (2007)).
} 
then the boundary condition implies $\Pi_{i}\left(x_{1}, x_{-1}\right)>0$ as otherwise the gradient field would not point inwards at $x$. Similarly, if $x_{1 i}=\bar{S}_{i}$ then $\Pi_{i}\left(x_{1}, x_{-1}\right)<0$. Suppose now that $x^{*}=\left(x_{1}^{*}, x_{-1}^{*}\right)$ is an equilibrium with $x_{1}^{*} \in \partial S$. Hence for at least one $1 \leq i \leq k$ we must have $x_{1 i}^{*} \in\left\{0, \bar{S}_{i}\right\}$. If $x_{1 i}^{*}=0$ then by the Kuhn-Tucker necessary conditions we must have $\Pi_{i}\left(x_{1}^{*}, x_{-1}^{*}\right) \leq 0$ which contradicts the boundary condition. If $x_{1 i}^{*}=\bar{S}_{i}$ then $\Pi_{i}\left(x_{1}^{*}, x_{-1}^{*}\right) \geq 0$, a contradiction. Hence only interior equilibria exist which proves i). For ii) note that $E$ is bounded and because of the continuity of $\phi$ (see proposition 1 i)) also closed. Because $\operatorname{Det}(J(x)) \neq 0$ at every $x \in E$ all equilibrium points are locally unique by the inverse function theorem. As $E$ is compact and discrete (all points locally isolated) $E$ must be finite.

\subsection{Proof of corollary 1}

Suppose that $\varphi\left(x_{-g}\right) \in \operatorname{Int}(S)$. Then we must have $\nabla \Pi^{g}\left(x_{1}, \ldots, \varphi\left(x_{-g}\right), \ldots, x_{N}\right)=0$. Strong quasiconcavity of $\Pi^{g}$ and the fact that $\Pi^{g} \in C^{2}$ imply

$$
\nabla \Pi^{g}\left(x_{1}, \ldots, \varphi\left(x_{-g}\right), \ldots, x_{N}\right)=0 \Rightarrow \operatorname{Det}\left(D_{x_{g}}^{2}\left(\Pi^{g}\left(x_{1}, \ldots, \varphi\left(x_{-g}\right), \ldots, x_{N}\right)\right)\right) \neq 0
$$

where $D_{x_{g}}^{2}$ denotes the Jacobian of $\nabla \Pi^{g}$ with respect to $x_{g}$. Hence by the Implicit Function Theorem we may conclude that $\varphi\left(x_{-g}\right)$ is differentiable at $x_{-g}$.

\subsection{Proof of theorem 2}

The proofs of theorems 2 and 3 require the following lemma.

Lemma 1 Let $\psi \in C([0,1],[0, \bar{S}])$ with $\psi(0) \neq \psi(1)$ and

$$
\psi(t) \in(0, \bar{S}) \quad \Rightarrow \quad \psi \text { differentiable at } t
$$

Then:

(i) if $\psi(0)>\psi(1) \exists t^{\prime} \in(0,1)$ such that $-\psi^{\prime}\left(t^{\prime}\right) \geq \psi(0)-\psi(1)$

(ii) if $\psi(0)<\psi(1) \exists t^{\prime \prime} \in(0,1)$ such that $\psi^{\prime}\left(t^{\prime \prime}\right) \geq \psi(1)-\psi(0)$ 
Proof: I first proove (i).

Suppose that $\psi(0)>\psi(1)$. Hence $\psi(0)>0$ and $\psi(1)<\bar{S}$. Define $T \equiv \psi^{-1}(\{0, \bar{S}\})$.

Case 1: $T=\emptyset$

As $\psi$ is differentiable on $(0,1)$ and continuous on $[0,1]$ the MVT implies that $\exists t \in(0,1)$ such that $-\psi^{\prime}(t)=\psi(0)-\psi(1)$.

Case 2: $T \neq \emptyset$

Obviously, $T$ is bounded. Because $\{0, \bar{S}\}$ is closed and $\psi$ is continuous $T$ also is closed and thus a compact subset of $\mathbb{R}$. Hence the min and max of $T$ exist and are denoted by $\underline{\mathrm{t}}, \bar{t}$. The proof now is a case-by-case examination.

(a) $\psi(\underline{\mathrm{t}})=0$. Then $\psi$ is continuous on the perfect interval $[0, \underline{\mathrm{t}}]$ and differentiable on $(0, \underline{\mathrm{t}})$. Thus by the MVT $\exists t \in(0, \underline{\mathrm{t}})$ such that

$$
\psi^{\prime}(t)=\frac{\psi(0)-\psi(\underline{\mathrm{t}})}{-\underline{\mathrm{t}}} \leq \frac{\psi(0)-\psi(1)}{-1}
$$

(b) $\psi(\underline{\mathrm{t}})=\bar{S}$ and $\psi(\bar{t})=\bar{S}$. Then $\psi$ is continuous on the perfect interval $[\bar{t}, 1]$ and differentiable on $(\bar{t}, 1)$. By the MVT $\exists t \in(\bar{t}, 1)$ such that

$$
\psi^{\prime}(t)=\frac{\psi(\bar{t})-\psi(1)}{\bar{t}-1} \leq \frac{\psi(0)-\psi(1)}{-1}
$$

(c) $\psi(\underline{\mathrm{t}})=\bar{S}$ and $\psi(\bar{t})=0$. Define $A \equiv \psi^{-1}(\{\bar{S}\})$, which is a non-empty and compact set. Hence $\hat{t}=\max A$ exists. Consider $B \equiv[\hat{t}, 1] \cap \psi^{-1}(\{0\})$, which also is non-empty and compact. Let $\check{t}=\min B$. Hence $\psi$ is continuous on the perfect interval $[\hat{t}, \check{t}]$ and differentiable on $(\hat{t}, \check{t})$. Thus by the MVT $\exists t \in(\hat{t}, \check{t})$ such that

$$
\psi^{\prime}(t)=\frac{\psi(\hat{t})-\psi(\check{t})}{\hat{t}-\check{t}} \leq \frac{\psi(0)-\psi(1)}{-1}
$$

Hence (i) is true and (ii) follows from (i) by setting $\rho(z) \equiv \psi(1-t)$.

\section{$\underline{\text { Proof of theorem } 2}$}


Step 1: $N=2$

Suppose that $x_{1}^{a}, x_{2}^{a}$ are asymmetric equilibria with the property that $\varphi\left(x_{2}^{a}\right)=x_{1}^{a}$ and $\varphi\left(x_{1}^{a}\right)=x_{2}^{a}$. Let $\psi(t) \equiv \varphi^{1}\left(x_{1}^{a}+t\left(x_{2}^{a}-x_{1}^{a}\right)\right)$ for $t \in[0,1]$. Then $\psi(0)=x_{2}^{a}$ and $\psi(1)=x_{1}^{a}$. Because $\psi(0) \neq \psi(1)$ lemma 1 together with proposition 1 (i) and corollary 1 asserts that there exists $t^{\prime} \in(0,1)$ such that either $\psi^{\prime}\left(t^{\prime}\right) \leq \psi(1)-\psi(0)$ or $\psi^{\prime}\left(t^{\prime}\right) \geq \psi(1)-\psi(0)$. In the first case we get (by the chain rule) that $\partial \varphi \cdot(\psi(0)-\psi(1)) \leq \psi(1)-\psi(0)$ where $\psi(1)-\psi(0)<0$, which implies $\partial \varphi\left(x_{2}\right) \leq-1$ for some $x_{2} \in(0, \bar{S})$. In the second case we get $\partial \varphi \cdot(\psi(0)-\psi(1)) \geq \psi(1)-\psi(0)$ where $\psi(1)-\psi(0)>0$. Consequently, again $\partial \varphi\left(x_{2}\right) \leq-1$ for some $x_{2} \in(0, \bar{S})$.

Step 2: $N>2$

Suppose $x^{a} \in S^{N}$ is an asymmetric equilibrium. Because of symmetry we can set $x^{a}=$ $\left(x_{1}^{a}, x_{2}^{a}, x_{3}^{a}, \ldots, x_{N}^{a}\right)$ with $x_{1}^{a} \neq x_{2}^{a}$. Suppose players $g=1$ and $g=2$ play a parametrised two-player game where $X=\left(x_{3}, \ldots, x_{N}\right) \in S^{N-2}$ is an exogenous vector of parameters. If we choose $X=\left(x_{3}^{a}, \ldots, x_{N}^{a}\right)$ then $\left(x_{1}^{a}, x_{2}^{a}\right)$ as well as $\left(x_{2}^{a}, x_{1}^{a}\right)$ must be asymmetric equilibria of the parametrised two-player game. Thus, by step 1 , if an asymmetric equilibrium exists we can always find $X \in S^{N-2}$ and $x_{2} \in(0, \bar{S})$ such that $\partial \varphi\left(x_{2} ; X\right) \leq-1$ - which is the contraposition of theorem 2 .

\subsection{Proof of theorem 3}

The proof will make use of the following fact: If $r_{1}, r_{2}, s_{1}, s_{2} \in \mathbb{R}$ with $r_{1} \leq s_{1} \leq 0$ and $r_{2} \leq s_{2} \leq 0$ then $r_{1} r_{2} \geq s_{1} s_{2}$.

Let $N=2$ and suppose an asymmetric equilibrium $x_{1}^{a}=\left(x_{11}^{a}, x_{12}^{a}\right) \in S$ exists. Then because of symmetry $x_{2}^{a}=\left(x_{21}^{a}, x_{22}^{a}\right)$ also is an asymmetric equilibrium where $\varphi\left(x_{2}^{a}\right)=x_{1}^{a}$ and $\varphi\left(x_{1}^{a}\right)=x_{2}^{a}$ and $x_{1}^{a} \neq x_{2}^{a}$. Define $\psi_{1}\left(t_{1}\right) \equiv \varphi_{1}\left(x_{1}^{a}+t_{1}\left(x_{2}^{a}-x_{1}^{a}\right)\right)$ and $\psi_{2}\left(t_{2}\right) \equiv \varphi_{2}\left(x_{1}^{a}+t_{2}\left(x_{2}^{a}-x_{1}^{a}\right)\right)$ for $t_{1}, t_{2} \in[0,1]$. Then $\psi_{1}(0)=\varphi_{1}\left(x_{1}^{a}\right), \psi_{1}(1)=\varphi_{1}\left(x_{2}^{a}\right), \psi_{2}(0)=\varphi_{2}\left(x_{1}^{a}\right)$ and $\psi_{2}(1)=\varphi_{2}\left(x_{2}^{a}\right)$. Moreover, $\psi_{i} \in C\left([0,1],\left[0, \bar{S}_{i}\right]\right)$ for $i=1,2$ and also

$$
\psi_{i}\left(t_{i}\right) \in\left(0, \bar{S}_{i}\right) \quad \Rightarrow \quad \psi_{i} \text { differentiable at } t_{i} \text { for } \mathrm{i}=1,2
$$


by corollary 1 . Then, assuming that the corresponding partial derivative exist, by the chain rule we have for $i=1$ :

$$
\psi_{1}^{\prime}\left(t_{1}\right)=\alpha\left(x_{1}^{a}+t_{1}\left(x_{2}^{a}-x_{1}^{a}\right)\right)\left(\psi_{1}(0)-\psi_{1}(1)\right)+\beta\left(x_{1}^{a}+t_{1}\left(x_{2}^{a}-x_{1}^{a}\right)\right)\left(\psi_{2}(0)-\psi_{2}(1)\right)
$$

and for $i=2$ :

$$
\psi_{2}{ }^{\prime}\left(t_{2}\right)=\gamma\left(x_{1}^{a}+t_{2}\left(x_{2}^{a}-x_{1}^{a}\right)\right)\left(\psi_{1}(0)-\psi_{1}(1)\right)+\delta\left(x_{1}^{a}+t_{2}\left(x_{2}^{a}-x_{1}^{a}\right)\right)\left(\psi_{2}(0)-\psi_{2}(1)\right)
$$

Case 1: $\varphi_{i}\left(x_{1}^{a}\right)=\varphi_{i}\left(x_{2}^{a}\right)$ for $i=1,2$.

Suppose that $\varphi_{1}\left(x_{1}^{a}\right)=\varphi_{1}\left(x_{2}^{a}\right)$. Hence $\psi_{1}(0)=\psi_{1}(1)$. Then $\varphi_{2}\left(x_{1}^{a}\right) \neq \varphi_{2}\left(x_{2}^{a}\right)$. According to lemma 1 there exists $t^{\prime} \in(0,1)$ such that either $\psi_{2}^{\prime}\left(t^{\prime}\right) \leq \psi_{2}(1)-\psi_{2}(0)$ or $\psi_{2}^{\prime}\left(t^{\prime}\right) \geq \psi_{2}(1)-\psi_{2}(0)$. In the first case we get $\delta \cdot\left(\psi_{2}(0)-\psi_{2}(1)\right) \leq \psi_{2}(1)-\psi_{2}(0)$ where $\psi_{2}(1)-\psi_{2}(0)<0$, which implies that $\exists\left(x_{21}, x_{22}\right) \in \operatorname{Int}(S)$ such that $\delta\left(x_{21}, x_{22}\right) \leq-1$. In the second case we get $\delta \cdot\left(\psi_{2}(0)-\psi_{2}(1)\right) \geq \psi_{2}(1)-\psi_{2}(0)$ where $\psi_{2}(1)-\psi_{2}(0)>0$. Consequently, again $\exists\left(x_{21}, x_{22}\right) \in$ $\operatorname{Int}(S)$ such that $\delta\left(x_{21}, x_{22}\right) \leq-1$. Finally, if instead $\varphi_{1}^{2}\left(x_{1}^{a}\right)=\varphi_{1}^{2}\left(x_{2}^{a}\right)$ then a similar argument gives that $\alpha \leq-1$ for some $\left(x_{21}, x_{22}\right) \in \operatorname{Int}(S)$.

From now on suppose that $\varphi_{i}\left(x_{1}^{a}\right) \neq \varphi_{i}\left(x_{2}^{a}\right)$ for $i=1,2$ and define $m \equiv \frac{\psi_{2}(0)-\psi_{2}(1)}{\psi_{1}(0)-\psi_{1}(1)}$.

Case 2.1: $\varphi_{i}\left(x_{1}^{a}\right)>\varphi_{i}\left(x_{2}^{a}\right)$ for $i=1,2$.

Then we have $\psi_{i}(0)>\psi_{i}(1)$ for $i=1,2$ and $\exists t_{i} \in(0,1)$ such that $\psi_{i}{ }^{\prime}\left(t_{i}\right) \leq \psi_{i}(1)-\psi_{i}(0)$ which implies

$$
\begin{aligned}
& \alpha+m \beta \leq-1 \\
& \gamma \frac{1}{m}+\delta \leq-1
\end{aligned}
$$

Suppose that $\alpha, \delta \geq-1 \forall\left(x_{21}, x_{22}\right) \in \operatorname{Int}(S)$ for which the corresponding derivative exists. Then

$$
\begin{aligned}
& m \beta \leq-(1+\alpha) \leq 0 \\
& \gamma \frac{1}{m} \leq-(1+\delta) \leq 0
\end{aligned}
$$

and the above fact implies that $\exists\left(x_{21}, x_{22}\right),\left(x_{21}^{\prime}, x_{22}^{\prime}\right) \in \operatorname{Int}(S)$ such that

$$
\beta\left(x_{21}, x_{22}\right) \gamma\left(x_{21}^{\prime}, x_{22}^{\prime}\right) \geq\left(1+\alpha\left(x_{21}, x_{22}\right)\right)\left(1+\delta\left(x_{21}^{\prime}, x_{22}^{\prime}\right)\right)
$$


Case 2.2: $\varphi_{i}\left(x_{1}^{a}\right)<\varphi_{i}\left(x_{2}^{a}\right)$ for $i=1,2$.

Then we have $\psi_{i}(0)<\psi_{i}(1)$ for $i=1,2$ and $\exists t_{i} \in(0,1)$ such that $\psi_{i}{ }^{\prime}\left(t_{i}\right) \geq \psi_{i}(1)-\psi_{i}(0)$ which implies

$$
\begin{aligned}
& \alpha+m \beta \leq-1 \\
& \gamma \frac{1}{m}+\delta \leq-1
\end{aligned}
$$

and a similar argument as in case 2.1 shows that $\beta \gamma \geq(1+\alpha)(1+\delta)$ must be true if $\alpha, \delta \geq-1$.

Case 2.3: $\varphi_{1}\left(x_{1}^{a}\right)<\varphi_{1}\left(x_{2}^{a}\right)$ and $\varphi_{2}\left(x_{1}^{a}\right)>\varphi_{2}\left(x_{2}^{a}\right)$ for $i=1,2$.

Hence $\psi_{1}(0)<\psi_{1}(1)$ and $\psi_{2}(0)>\psi_{2}(1)$ and there exist $t_{1}, t_{2} \in(0,1)$ such that $\psi_{1}{ }^{\prime}\left(t_{1}\right) \geq$ $\psi_{1}(1)-\psi_{1}(0)$ and $\psi_{2}^{\prime}\left(t_{2}\right) \leq \psi_{2}(1)-\psi_{2}(0)$. Consequently, we get

$$
\begin{aligned}
& m \beta \leq-(1+\alpha) \leq 0 \\
& \gamma \frac{1}{m} \leq-(1+\delta) \leq 0
\end{aligned}
$$

and a similar argument as in case 2.1 shows that $\beta \gamma \geq(1+\alpha)(1+\delta)$ must be true if $\alpha, \delta \geq-1$.

Case 2.4: $\varphi_{1}\left(x_{1}^{a}\right)>\varphi_{1}\left(x_{2}^{a}\right)$ and $\varphi_{2}\left(x_{1}^{a}\right)<\varphi_{2}\left(x_{2}^{a}\right)$ is treated similarly to case 2.3 .

Thus if an asymmetric equilibrium exists we can always find interior points such that $\alpha \leq-1$, $\delta \leq-1$ or $(1+\alpha)(1+\delta) \leq \beta \gamma$ and the statement of theorem 3 simply is the contraposition of this result.

The proof is concluded by noting that for $N>2$ if condition (15) holds for any given parameter vector $X \in S^{N-2}$ then, by the proof of theorem 2 , there cannot be any asymmetric equilibrium in the game.

Remark: By defining

$$
A \equiv\left(\begin{array}{cc}
1+\frac{\partial \varphi_{1}}{\partial x_{21}} & \frac{\partial \varphi_{1}}{\partial x_{22}} \\
\frac{\partial \varphi_{2}}{\partial x_{21}} & 1+\frac{\partial \varphi_{2}}{\partial x_{22}}
\end{array}\right)
$$

condition (15) of theorem 3 can be compactly written as the requirement that all principal minors of $A$ be equal or greater than zero. Theorem 3 naturally can be extended to the case where $k>2$. 
To illustrate this suppose that $N=2$ and $x_{1}^{a}$ and $x_{2}^{a}$ are two different asymmetric equilibria with $\varphi\left(x_{1}^{a}\right)=x_{2}^{a}$ and $\varphi\left(x_{2}^{a}\right)=x_{1}^{a}$. For simplicity, assume that $\varphi\left(x_{1}^{a}+t\left(x_{2}^{a}-x_{1}^{a}\right)\right) \in \operatorname{Int}(S)$ for $t \in(0,1)$. Then the mean value theorem asserts the existence of $k$ vectors $x_{2}^{i} \in \operatorname{Int}(S)$, $1 \leq i \leq k$, such that

$$
\tilde{A}\left(\begin{array}{c}
\Delta_{1} \\
\vdots \\
\Delta_{k}
\end{array}\right)=-\left(\begin{array}{c}
\Delta_{1} \\
\vdots \\
\Delta_{k}
\end{array}\right)
$$

where $\tilde{A}$ is a $k \times k$-matrix with $a_{i j}=\frac{\partial \varphi_{i}\left(x_{2}^{i}\right)}{\partial x_{2 j}}$ and $\Delta_{i} \equiv x_{2 i}^{a}-x_{1 i}^{a}$. But (26) is equivalent to

$$
(I+\tilde{A})\left(\begin{array}{c}
\Delta_{1} \\
\vdots \\
\Delta_{k}
\end{array}\right)=A\left(\begin{array}{c}
\Delta_{1} \\
\vdots \\
\Delta_{k}
\end{array}\right)=0
$$

where

$$
A=\left(\begin{array}{cccc}
1+\frac{\partial \varphi_{1}}{\partial x_{21}} & \frac{\partial \varphi_{1}}{\partial x_{22}} & \cdots & \frac{\partial \varphi_{1}}{\partial x_{2 k}} \\
\frac{\partial \varphi_{2}}{\partial x_{21}} & 1+\frac{\partial \varphi_{2}}{\partial x_{22}} & \cdots & \frac{\partial \varphi_{2}}{\partial x_{2 k}} \\
\vdots & \vdots & \ddots & \vdots \\
\frac{\partial \varphi_{k}}{\partial x_{21}} & \cdots & \cdots & 1+\frac{\partial \varphi_{k}}{\partial x_{2 k}}
\end{array}\right)
$$

Because $x_{1}^{a}$ and $x_{2}^{a}$ are different asymmetric equilibria we must have $\Delta_{i} \neq 0$ for at least one $i$. Consequently, we must have $\operatorname{rank}(A)<k$ in $(27)$ and hence $\operatorname{Det}(A)=0$. Now suppose that $\Delta_{k}=0$. Then (27) implies that

$$
A_{k-1}\left(\begin{array}{c}
\Delta_{1} \\
\vdots \\
\Delta_{k-1}
\end{array}\right)=0
$$

where $A_{k-1}$ is formed from $A$ by cancelling the $k$-th row and column. Hence $\operatorname{Det}\left(A_{k-1}\right)=0$, where $\operatorname{Det}\left(A_{k-1}\right)$ is a principal minor of order $k-1$ of $A$. Obviously, if $\Delta_{j}=0$ for any $j=1, \ldots, k$ then the corresponding principal minor of order $k-1$ of $A$ must be zero. This argument may be continued up to the case that $k-1$ of the $k \Delta_{i}$ 's are zero and we find that this corresponds to the fact that at least one principal minor of $A$ is equal to zero if an asymmetric equilibrium exists. Consequently, if we can show that all principal minors of $A$ are positive we may conclude that no interior asymmetric equilibria nor asymmetric equilibria on the boundary 
with $\varphi\left(x_{1}^{a}+t\left(x_{2}^{a}-x_{1}^{a}\right)\right) \in \operatorname{Int}(S)$ for $t \in(0,1)$ can exist. A somewhat tedious case-by-case examination as in the proof of theorem 3 shows that if $\varphi\left(x_{1}^{a}+t\left(x_{2}^{a}-x_{1}^{a}\right)\right) \notin \operatorname{Int}(S)$ for $t \in(0,1)$ we can always find $x_{2}^{1}, \ldots, x_{2}^{k} \in \operatorname{Int}(S)$ such that at least one principal minor of $A$ is equal or less than zero.

\subsection{Proof of proposition 4}

(a) Applying (12) to (16) gives (17).

(b) From

$$
\Pi_{1}\left(q_{1}, q_{2} ; q_{3}, \ldots, q_{N}\right)=P\left(q_{1}+\sum_{j=2}^{n} q_{j}\right)+q_{1} P^{\prime}\left(q_{1}+\sum_{j=2}^{n} q_{j}\right)-c^{\prime}\left(q_{1}\right)=0
$$

we get

$$
\frac{\Pi_{12}}{\Pi_{11}}=\frac{P^{\prime}(Q)+q_{1} P^{\prime \prime}(Q)}{2 P^{\prime}(Q)+q_{1} P^{\prime \prime}(Q)-c^{\prime \prime}\left(q_{1}\right)}
$$

and the claim follows from corollary 2 .

(c) Obvious.

\subsection{Proof of proposition 5}

The proof of proposition 8 requires the following lemma.

Lemma 2 (Characterisation of asymmetric equilibria) In a symmetric one-dimensional two-player game no asymmetric equilibria exist if and only if

$$
\varphi^{1}\left(\varphi^{2}\left(x_{1}\right)\right)>x_{1} \quad \forall x_{1} \in S \text { with } \varphi^{2}\left(x_{1}\right)>x_{1} .
$$

Proof:

$" \Rightarrow "$. If $\left(x_{1}, x_{2}\right)$ is an asymmetric equilibrium then $x_{1} \neq x_{2}$ and $\varphi^{1}\left(\varphi^{2}\left(x_{1}\right)\right)=x_{1}$. Hence if $(28)$ holds then no asymmetric equilibrium can exist. 
$" \Leftarrow "$. The proof of this direction naturally is more involved and requires the following additional notation. Let $G_{1} \equiv\left\{\left(x_{1}, x_{2}\right) \in S^{2}: \varphi^{1}\left(x_{2}\right)=x_{1}\right\}$ and $G_{2} \equiv\left\{\left(x_{1}, x_{2}\right) \in S^{2}: \varphi^{2}\left(x_{1}\right)=x_{2}\right\}$ denote the graphs of the best-response functions of the two players. Further, $G_{1}\left(x_{2}\right) \equiv\left(\varphi^{1}\left(x_{2}\right), x_{2}\right)$ and $G_{2}\left(x_{1}\right) \equiv\left(x_{1}, \varphi^{2}\left(x_{1}\right)\right)$ deonze specific points on the graphs. Define the lower and the upper area of the graph of player $j=2$ as

$$
\begin{aligned}
& G_{2}^{L} \equiv\left\{\left(x_{1}, x_{2}\right) \in S^{2}: x_{2} \leq \varphi^{2}\left(x_{1}\right)\right\} \\
& G_{2}^{U} \equiv\left\{\left(x_{1}, x_{2}\right) \in S^{2}: x_{2} \geq \varphi^{2}\left(x_{1}\right)\right\}
\end{aligned}
$$

Note that $G_{2}^{L}, G_{2}^{U}$ are closed in $S^{2}$ as $G_{2}\left(x_{1}\right)$ is a continuous function.

Suppose that $\exists \hat{x}_{1} \in S$ with $\varphi^{2}\left(\hat{x}_{1}\right) \equiv \hat{x}_{2}>\hat{x}_{1}$ and $\varphi^{1}\left(\varphi^{2}\left(\hat{x}_{1}\right)\right) \leq \hat{x}_{1}$. If $\varphi^{1}\left(\varphi^{2}\left(\hat{x}_{1}\right)\right)=\hat{x}_{1}$ there is nothing to proof so suppose that $\varphi^{1}\left(\varphi^{2}\left(\hat{x}_{1}\right)\right)<\hat{x}_{1}$. Let $R \subset S^{2}$ be definde by $R \equiv S \times\left[0, \hat{x}_{1}\right]$.

Case 1: $E^{s} \cap R=\emptyset$.

If $G_{1}\left(\hat{x}_{2} \in \operatorname{Int}\left(G_{2}^{U}\right)\right.$ then as $G_{1}(0) \in G_{2}^{L}$ and $G_{1}\left(x_{2}\right)$ as well as $G_{2}\left(x_{1}\right)$ are continuous functions $\exists x_{2} \in\left(0, \hat{x}_{2}\right)$ such that $G_{1}\left(x_{2}\right) \in G_{2} \mid R$. Hence an intersection of $G_{1}$ and $G_{2}$ in $R$ exists which, by presupposition, cannot be symmetric.

If $G_{1}\left(\hat{x}_{2} \in \operatorname{Int}\left(G_{2}^{L}\right)\right.$ then as $G_{1}(\bar{S}) \in G_{2}^{U} \exists x_{2} \in\left(\hat{x}_{2}, \bar{S}\right]$ such that $G_{1}\left(x_{2}\right) \in G_{2} \mid R$. Again, this intersection must be an asymmetric equilibrium.

Case 2: $E^{s} \cap R \neq \emptyset$.

Let $x^{*} \equiv \max \left\{E^{s} \cap R\right\}$. We always have $x^{s}<\hat{x}_{1}$. Because $\varphi^{2}\left(\hat{x}_{1}\right)>\hat{x}_{1}$ we can assume wlog that

$$
\varphi^{2}\left(x_{1}\right)>x_{1} \quad \forall x_{1} \in\left(x^{s}, \hat{x}_{1}\right)
$$

as otherwise by the continuity of $G_{2}\left(x_{1}\right)$ and $\varphi^{2}\left(\hat{x}_{1}\right)>\hat{x}_{1}$ together imply the existence of a further symmetric equilibrium in $\left\{E^{s} \cap R\right\}$ contradicting the maximality of $x^{s}$.

By symmetry and continuity of the best-response function, we can find a (sufficiently small) $\varepsilon>0$ such that $\varphi^{1}\left(x^{s}+\varepsilon\right)>x^{s}+\varepsilon$ but $\varphi^{1}\left(x^{s}+\varepsilon\right)<\hat{x}_{1}$. But then (29) implies

$$
\varphi^{2}\left(\varphi^{1}\left(x^{s}+\varepsilon\right)\right)>\varphi^{1}\left(x^{s}+\varepsilon\right)>x^{s}+\varepsilon
$$


Hence $G_{1}\left(x^{s}+\varepsilon\right) \in \operatorname{Int}\left(G_{2}^{L}\right)$. But then the same argumentation as in case 1 immediately shows that $\exists x_{2} \in\left(x^{s}, \bar{S}\right)$ such that $G_{1}\left(x_{2}\right) \in G_{2} \mid R$. Thus a further intersection of the graphs must exist which cannot be symmetric.

Proof of proposition 5:

i) If the binary symmetric game with $c_{g}=c_{g+1}=\underline{\mathrm{c}}$ has no asymmetric equilibria for a given $X_{g}$ then because of lemma 2

$$
\varphi^{g}\left(\varphi^{g+1}\left(x_{g} ; X_{g}, \underline{\mathrm{c}}\right) ; X_{g}, \underline{\mathrm{c}}\right)>x_{g} \quad \text { for } x_{g} \in S \text { with } \varphi^{g+1}\left(x_{g} ; X_{g}, \underline{\mathrm{c}}\right)>x_{g}
$$

But then the monotonicity property implies that $\forall c \in(\underline{c}, \bar{c}]$ we must have

$$
\varphi^{g}\left(\varphi^{g+1}\left(x_{g} ; X_{g}, \underline{\mathrm{c}}\right) ; X_{g}, c\right) \geq \varphi^{g}\left(\varphi^{g+1}\left(x_{g} ; X, \underline{\mathrm{c}}\right) ; X_{g}, \underline{\mathrm{c}}\right)
$$

Because of (30) this gives

$$
\varphi^{g}\left(\varphi^{g+1}\left(x_{g} ; X_{g}, \underline{\mathrm{c}}\right) ; X_{g}, c\right)>x_{g}
$$

for $\varphi^{g+1}\left(x_{g} ; X_{g}, \underline{\mathrm{c}}\right)>x_{g}$. Thus for any given $X_{g}$ the binary asymmetric game can only have equilibria with $x_{g}\left(X_{g}, c_{g}\right) \geq x_{g+1}\left(X_{g}, c_{g+1}\right)$. As this statement must hold for any $1 \leq g \leq N-1$ the asymmetric game can only have equilibria with $x_{1}^{*} \geq x_{2}^{*} \geq, \ldots, \geq x_{N}^{*}$.

ii) Because of i) we have $x_{1}^{*} \geq x_{2}^{*} \geq, \ldots, \geq x_{N}^{*}$. Strict monotonicity means that

$$
c^{\prime}>c \quad \Rightarrow \quad \varphi^{g}\left(x_{-g}, c^{\prime}\right)>\varphi^{g}\left(x_{-g}, c\right)
$$

for any $c, c^{\prime} \in[\underline{\mathrm{c}}, \bar{c}]$. Now suppose that $x^{*}$ is an equilibrium of the asymmetric game such that $x_{g}^{*}=x_{g+1}^{*}=x_{g}^{s}$. Hence we must have

$$
\varphi^{g}\left(x_{g}^{s} ; X_{g}^{*}, c_{g}\right)=\varphi^{g+1}\left(x_{g}^{s} ; X_{g}^{*}, c_{g+1}\right)=\varphi^{g}\left(x_{g}^{s} ; X_{g}^{*}, c_{g+1}\right)
$$

which contradicts $(31)$ as $c_{g}>c_{g+1}$. 
iii) From ii) we have $x_{1}^{*}>x_{2}^{*}>\ldots>x_{N}^{*}$. If we consider the asymmetric binary game between players $N-1$ and $N$ for given $X_{N-1}^{*}$ we see that because

$$
\varphi^{N-1}\left(x^{s}(\underline{\mathrm{c}}) ; X_{N-1}^{*}, c_{N-1}\right)>\varphi^{N-1}\left(x^{s}(\underline{\mathrm{c}}) ; X_{N-1}^{*}, \underline{\mathrm{c}}\right)
$$

$x^{s}(\underline{\mathrm{c}})$ cannot be an equilibrium of this game. Hence we must have $x_{N-1}^{*}>x^{s}(\underline{\mathrm{c}})$.

\subsection{Proof of proposition 6}

Suppose that $x^{*} \in E^{s}$ is localy symmetrically stable. For simplicity, the norm in use is $\|\cdot\|_{\infty}$ but any other norm also works. Thus $\exists \varepsilon>0$ such that $\forall x_{1}(0) \in \mathbb{B}\left(x_{1}^{*}, \varepsilon\right)$ we have that $x_{1}(t) \rightarrow x_{1}^{*}$. Hence if $x(0) \in M$ we have $x(0)=\left(x_{1}(0), \ldots, x_{1}(0)\right)$ and $x_{1}(0) \in \mathbb{B}\left(x_{1}^{*}, \varepsilon\right)$. Consequently, $\forall$ $x(0) \in M$ we have that $x(t) \rightarrow x^{*}$.

The spectrum of $\hat{J}\left(x_{1}^{*}\right)$ is a subset of the spectrum of $\hat{J}\left(x^{*}\right)$. To see this suppose that $\lambda$ is an eigenvalue of $\hat{J}\left(x_{1}^{*}\right)$, i.e. $\hat{J}\left(x_{1}^{*}\right) \hat{c}=\lambda \hat{c}$ for a $\hat{c} \neq 0$. Then $\lambda$ also is eigenvalue of $\hat{J}\left(x^{*}\right)$ iff $D \hat{J}\left(x^{*}\right) c=\lambda D c$ for a $c \neq 0$ and any given $N k \times N k$ matrix $D$. Note that $\hat{J}\left(x^{*}\right)$ takes on the form

$$
\hat{J}\left(x^{*}\right)=\left(\begin{array}{ccccc}
F & \bar{F} & \bar{F} & \ldots & \bar{F} \\
\bar{F} & F & \bar{F} & \ldots & \bar{F} \\
\ldots & \ldots & \ldots & \ldots & \ldots \\
\ldots & \ldots & \ldots & \ldots & \ldots \\
\bar{F} & \bar{F} & & \bar{F} & F
\end{array}\right)
$$

where $F \equiv \frac{\partial^{2} \Pi^{1}\left(x^{*}\right)}{\partial x_{1} \partial x_{1}^{T}}$ denotes the Hessian of $\Pi^{1}$ with respect to $x_{1}$ and $\bar{F} \equiv \frac{\partial^{2} \Pi^{1}\left(x^{*}\right)}{\partial x_{1} \partial x_{g}^{T}}$ is the matrix of second derivatives of $\Pi^{1}$ with respect to $x_{g}$ with $g \neq 1$. But if $D$ is the partioned matrix

$$
D=\left(\begin{array}{ccc}
I_{k} & \cdots & I_{k} \\
\cdots & \cdots & \cdots \\
I_{k} & \cdots & I_{k}
\end{array}\right)
$$


then $D \hat{J}\left(x^{*}\right)$ is a partitioned matrix with $F+(N-1) \bar{F}$ in every partition. But as $F+(N-1) \bar{F}=$ $\hat{J}\left(x_{1}^{*}\right)$ we have

$$
D \hat{J}\left(x^{*}\right) c=\lambda D c \quad c=\left(\begin{array}{c}
\hat{c} \\
0 \\
\vdots \\
0
\end{array}\right) \neq 0
$$

Hence $\lambda$ also belongs to the spectrum of $\hat{J}\left(x^{*}\right)$.

\subsection{Proof of proposition 7}

i) Let $E_{1} \equiv\left\{x_{1} \in \operatorname{Int}(S): \nabla \tilde{\Pi}\left(x_{1}\right)=0\right\}$ and suppose that $E_{1}$ is multi-valued. Then because of proposition 3 there must exist $x_{1} \in E_{1}$ such that $\operatorname{Det}\left(-\tilde{J}\left(x_{1}\right)\right)<0$. Because $\operatorname{Det}\left(-\hat{J}\left(x_{1}\right)\right)=s_{1} \cdot \ldots \cdot s_{k} \cdot \operatorname{Det}\left(-\tilde{J}\left(x_{1}\right)\right)$ we also have $\operatorname{Det}\left(-\hat{J}\left(x_{1}\right)\right)<0$. Let $\lambda_{1}, \ldots, \lambda_{k}$ denote the eigenvalues of $-\hat{J}\left(x_{1}\right)$. Then $\prod_{i=1}^{k} \lambda_{k}<0$, which implies that there must exist at least one negative eigenvalue. Consequently, $\hat{J}\left(x_{1}\right)$ must have at least one positive eigenvalue which means that $x=\left(x_{1}, \ldots, x_{1}\right)$ is an unstable equilibrium.

ii) Inexistence of multiple symmetric equilibria is obvious. Let $\lambda_{1}, \lambda_{2}$ denote the eigenvalues of $\hat{J}\left(x_{1}\right)$. Then, because $s_{1}, s_{2}>0,(24)$ implies:

$$
\begin{aligned}
& \lambda_{1}+\lambda_{2}=\operatorname{Trace}\left(\hat{J}\left(x_{1}\right)\right)=s_{1} \tilde{\Pi}_{11}+s_{2} \tilde{\Pi}_{22}<0 \\
& \lambda_{1} \lambda_{2}=\operatorname{Det}\left(\hat{J}\left(x_{1}\right)\right)=s_{1} s_{2} \operatorname{Det}\left(\tilde{J}\left(x_{1}\right)\right)>0
\end{aligned}
$$

which implies that $\lambda_{1}, \lambda_{2}$ must either be negative or have negative real parts.

iii) $\quad($ a $) \Leftrightarrow\left(\right.$ b) follows as $\operatorname{Det}\left(\hat{J}\left(x_{1}\right)\right), \operatorname{Det}\left(\tilde{J}\left(x_{1}\right)\right)<0$ holds iff $\tilde{\Pi}_{11}\left(x_{1}\right)<0$ for $x_{1} \in E_{1}$.

To see $(\mathrm{b}) \Leftrightarrow(\mathrm{c})$ note that from $\tilde{\Pi}_{1}\left(x_{1}, \bar{x}, c\right)=0$ we have that

$$
x_{1}{ }^{\prime}(c)=-\frac{\frac{\partial}{\partial c} \tilde{\Pi}_{1}}{\Pi_{11}}-\frac{\Pi_{12}(N-1)}{\Pi_{11}} \bar{x}^{\prime}(c)
$$

where the first term is the direct effect and the second term the strategic effect. Because 
in equilibrium $\left(x_{1}^{\prime}(c)=\bar{x}^{\prime}(c)\right)$

$$
x_{1}^{\prime}(c)=-\frac{\frac{\partial}{\partial c} \tilde{\Pi}_{1}}{\Pi_{11}} \frac{\Pi_{11}}{\Pi_{11}+(N-1) \Pi_{12}}=-\frac{\frac{\partial}{\partial c} \tilde{\Pi}_{1}}{\tilde{\Pi}_{11}}
$$

we see that $\operatorname{sign}\left(x_{1}{ }^{\prime}(c)\right)=\operatorname{sign}\left(\frac{\partial}{\partial c} \tilde{\Pi}_{1}\right)$, i.e. strategic effects are of second-order importance, iff $\tilde{\Pi}_{11}\left(x_{1}\right)<0$.

To see the last claim note that a symmetric equilibrium point $x$ is locally stable under (general) myopic adjustments iff the symmetric $N \times N$-matrix

$$
\mathrm{J}(\mathrm{x})=\left(\begin{array}{ccccc}
\Pi_{11} & \Pi_{12} & \Pi_{12} & & \Pi_{12} \\
\Pi_{12} & \Pi_{11} & \Pi_{12} & \cdots & \Pi_{12} \\
\cdots & \cdots & \cdots & \cdots & \cdots \\
\cdots & \cdots & \cdots & \cdots & \cdots \\
\Pi_{12} & \Pi_{12} & \cdots & \Pi_{12} & \Pi_{11}
\end{array}\right)
$$

is negative definite. This is the case iff

$$
\Pi_{11}(x)<\Pi_{12}(x)
$$

and

$$
\Pi_{11}(x)+(N-1) \Pi_{12}(x)=\tilde{\Pi}_{11}\left(x_{1}\right)<0
$$

which holds by presupposition.

\subsection{Proof of proposition 8}

Suppose that $x_{1} \in E_{1}$. If $\hat{M}\left(x_{1}\right)$ is positive definite $\forall s_{1}, \ldots, s_{k}>0$ then i) all eigenvalues of $-\hat{J}\left(x_{1}\right)$ must be positive or have positive real part (see e.g. Tang et al. (2007)) and ii) $-\hat{J}\left(x_{1}\right)$ is a $P$-matrix (see e.g. Gale and Nikaido (1965), p. 84). But i) implies that $\hat{J}\left(x_{1}\right)$ only has negative eigenvalues or eigenvalues with negative real parts. Hence $x=\left(x_{1}, \ldots, x_{1}\right)$ must be a symmetrically stable equilibrium. Further, ii) implies that $\operatorname{Det}\left(-\hat{J}\left(x_{1}\right)\right)>0$ and the second 
claim follows from $\operatorname{Det}\left(-\hat{J}\left(x_{1}\right)\right)=s_{1} \cdot \ldots \cdot s_{k} \operatorname{Det}\left(-\tilde{J}\left(x_{1}\right)\right)$.

\subsection{Proof of proposition 9}

The supermodularity together with the positivity of the principal minors and $s_{1}, \ldots, s_{k}>0$ imply that $-\hat{J}\left(x_{1}\right)$ is an M-Matrix (a matrix with non-positive off diagonal elements, positive diagonal elements and positive principal minors) for any choice of $s_{1}, \ldots, s_{k}>0$. But as all eigenvalues of an M-Matrix are known to be positive or have positive real part (see Tang et al. (2007)) the claim follows immediately.

\subsection{Proof of theorem 4}

The proof of theorem 4 requires the following two lemmata. The norm in use is $\|\cdot\| \equiv\|\cdot\|_{\infty}$. Let $\overline{\mathbb{B}}\left(x^{*}, \varepsilon\right)$ denote a closed $\varepsilon$-ball about $x^{*}$.

Lemma 3 Suppose $x^{*} \in E^{s}$ and let $\phi$ be the joint best-response function (4) and $V \equiv \overline{\mathbb{B}}\left(x^{*}, \varepsilon\right) \subset$ $S^{N}$. Then $\phi: V \rightarrow S^{N}$ is a contraction on $V$ if $\varphi_{i}: \bar{U}_{1} \rightarrow S_{i}$ for $\bar{U}_{1} \equiv \overline{\mathbb{B}}\left(x_{-1}^{*}, \varepsilon\right) \subset S^{N-1}$ is a contraction on $\bar{U}_{1}$ for $i=1, \ldots, k$.

\section{Proof:}

Because of symmetry we need only regard player $g=1$. Suppose that for every $1 \leq i \leq k \varphi_{i}$ is a contraction on $\bar{U}_{1}$ for a given $\varepsilon>0$. Hence there exists $q_{i} \in[0,1)$ with

$$
x_{-1} \in \bar{U}_{1} \quad \Rightarrow \quad\left|\varphi_{i}\left(x_{-1}^{*}\right)-\varphi_{i}\left(x_{-1}\right)\right| \leq q_{i}\left\|x_{-1}^{*}-x_{-1}\right\|
$$

Let $q \equiv \max \left\{q_{i}\right\}$ and $x_{-1} \in \bar{U}_{1}$. Then

$$
\begin{aligned}
\left\|\varphi\left(x_{-1}^{*}\right)-\varphi\left(x_{-1}\right)\right\| & =\max _{1 \leq i \leq k}\left\{\left|\varphi_{i}\left(x_{-1}^{*}\right)-\varphi_{i}\left(x_{-1}\right)\right|\right\} \\
& \leq q\left\|x_{-1}^{*}-x_{-1}\right\|
\end{aligned}
$$


Hence $\varphi$ is a contraction on $\bar{U}_{1}$. By symmetry $\varphi\left(x_{-g}\right)$ is a contraction on $\bar{U}_{g} \equiv \overline{\mathbb{B}}\left(x_{-g}^{*}, \varepsilon\right)$ for $g=2, \ldots, N$. Suppose $x \in V$ and hence $x_{-g} \in \bar{U}_{g}$. Then

$$
\begin{aligned}
\left\|\phi\left(x^{*}\right)-\phi(x)\right\| & \leq q\left\|x_{-g}^{*}-x_{-g}\right\| \quad g=1, \ldots, N \\
& \leq q\left\|x^{*}-x\right\|
\end{aligned}
$$

which shows that $\phi(x)$ is a contraction on $V$.

Lemma 4 Suppose that $\sum_{j=1}^{k}\left|\frac{\partial \varphi_{i}\left(x_{-1}^{*}\right)}{\partial x_{2 j}}\right|<\frac{1}{N-1}$ and $x^{*} \in E^{s}$. Then there exists a closed ball $\bar{U}_{i} \subset S^{N-1}$ about $\left(x_{-1}^{*}\right)$ such that $\varphi_{i}$ is a contraction on $\bar{U}_{i}$.

\section{Proof:}

Define $\sigma_{i}\left(x_{-1}\right) \equiv \sum_{j=1}^{k}\left|\frac{\partial \varphi_{i}\left(x_{-1}\right)}{\partial x_{2 j}}\right|$ and $q \equiv(N-1) \sigma_{i}\left(x_{-1}^{*}\right)$. Then $q \in[0,1)$. As $\sigma_{i}\left(x_{-1}\right)$ is continuous on $S^{N-1}$ we have that for all $\varepsilon \in\left(0, \frac{1-q}{N-1}\right]$ there exists a $\delta(\varepsilon)>0$ such that

$$
\left\|x_{-1}^{*}-x_{-1}\right\| \leq \delta \Rightarrow\left|\sigma_{i}\left(x_{-1}^{*}\right)-\sigma_{i}\left(x_{-1}\right)\right|<\varepsilon
$$

Hence if $\sigma_{i}\left(x_{-1}^{*}\right)>\sigma_{i}\left(x_{-1}\right)$ then $\sigma_{i}\left(x_{-1}\right)<\frac{q}{N-1}<\frac{1}{N-1}$. If $\sigma_{i}\left(x_{-1}^{*}\right) \leq \sigma_{i}\left(x_{-1}\right)$ then

$$
\sigma_{i}\left(x_{-1}\right)<\varepsilon+\sigma_{i}\left(x_{-1}^{*}\right)=\varepsilon+\frac{q}{N-1} \leq \frac{1}{N-1}
$$

Let $\delta \equiv \delta\left(\frac{1-q}{N-1}\right)$ and $\bar{U}_{i} \equiv \overline{\mathbb{B}}\left(\left(x_{-1}^{*}\right), \delta\right)$. Hence

$$
x_{-1} \in \bar{U}_{i} \quad \Rightarrow \quad \sigma_{i}\left(x_{-1}\right)<\frac{1}{N-1}
$$

Because of symmetry we have

$$
x_{-1} \in \bar{U}_{i} \quad \Rightarrow \quad \sum_{j=1}^{k}\left|\frac{\partial \varphi_{i}\left(x_{-1}\right)}{\partial x_{n j}}\right|<\frac{1}{N-1} \quad \forall n=2, \ldots, N
$$


By the mean value theorem:

$$
\left|\varphi_{i}\left(x_{-1}^{\prime}\right)-\varphi_{i}\left(x_{-1}\right)\right| \leq\left\|x_{-1}^{\prime}-x_{-1}\right\| \sup _{\substack{0 \leq t \leq 1 \\\left\|\tilde{x}_{-1}\right\|=1}}\left|\nabla \varphi_{i}\left(x_{-1}{ }^{\prime}+t\left(x_{-1}-x_{-1}^{\prime}\right)\right) \cdot\left(\tilde{x}_{-1}\right)^{T}\right|
$$

where $x_{-1}, x_{-1}^{\prime} \in \bar{U}_{i}$ imply that also $x_{-1}+t\left(x_{-1}^{\prime}-x_{-1}\right) \in \bar{U}_{i}$ for $t \in[0,1]$ as the closed ball $\bar{U}_{i}$ is a convex set. Let $x(t) \equiv x_{-1}{ }^{\prime}+t\left(x_{-1}-x_{-1}{ }^{\prime}\right)$. Then

$$
\begin{aligned}
Q & \equiv \sup _{\substack{0 \leq t \leq 1 \\
\left\|\tilde{\tilde{x}}_{-1}\right\|=1}}\left|\nabla \varphi_{i}(x(t)) \cdot\left(\tilde{x}_{-1}\right)\right| \\
& =\sup _{\substack{0 \leq t \leq 1 \\
\left\|\tilde{x}_{-1}\right\|=1}}\left|\frac{\partial \varphi_{i}}{\partial x_{21}}(x(t)) \tilde{x}_{21}+\frac{\partial \varphi_{i}}{\partial x_{22}}(x(t)) \tilde{x}_{22}+\ldots+\frac{\partial \varphi_{i}}{\partial x_{N k}}(x(t)) \tilde{x}_{N k}\right| \\
& =\sup _{0 \leq t \leq 1}\left\{\left|\frac{\partial \varphi_{i}}{\partial x_{21}}(x(t))\right|+\ldots+\left|\frac{\partial \varphi_{i}}{\partial x_{2 k}}(x(t))\right|+\ldots+\left|\frac{\partial \varphi_{i}}{\partial x_{N 1}}(x(t))\right|+\ldots+\left|\frac{\partial \varphi_{i}}{\partial x_{N k}}(x(t))\right|\right\} \\
& =\left|\frac{\partial \varphi_{i}}{\partial x_{21}}\left(t^{*}\right)\right|+\ldots+\left|\frac{\partial \varphi_{i}}{\partial x_{2 k}}\left(x\left(t^{*}\right)\right)\right|+\ldots+\left|\frac{\partial \varphi_{i}}{\partial x_{N 1}}\left(x\left(t^{*}\right)\right)\right|+\ldots+\left|\frac{\partial \varphi_{i}}{\partial x_{N k}}\left(x\left(t^{*}\right)\right)\right|
\end{aligned}
$$

where the last step follows from the continuity of the partial derivatives and $t \in[0,1]$.

But (32) then implies

$$
Q<\frac{N-1}{N-1}=1
$$

As a consequence

$$
\left|\varphi_{i}\left(x_{-1}^{\prime}\right)-\varphi_{i}\left(x_{-1}\right)\right| \leq Q\left\|x_{-1}^{\prime}-x_{-1}\right\|
$$

for all $x_{-1}, x_{-1}^{\prime} \in \bar{U}_{i}$ and $Q \in[0,1)$. Hence $\varphi_{i}$ is a contraction on $\bar{U}_{i}$.

\section{Proof of theorem 4:}

Because of permutation-symmetry it does not matter which player marginally changes his strategy at a symmetric equilibrium. Then for $j=1, \ldots, k$

$$
\left|\frac{\partial \tilde{\varphi}_{i}\left(\bar{x}^{*}\right)}{\partial \bar{x}_{j}}\right|=(N-1)\left|\frac{\partial \varphi_{i}\left(x_{-1}^{*}\right)}{\partial x_{2 j}}\right|
$$


Hence

$$
\sum_{j=1}^{k}\left|\frac{\partial \tilde{\varphi}_{i}\left(\bar{x}^{*}\right)}{\partial \bar{x}_{j}}\right|=(N-1) \sum_{j=1}^{k}\left|\frac{\partial \varphi_{i}\left(x_{-1}^{*}\right)}{\partial x_{2 j}}\right|
$$

and thus by presupposition

$$
\sum_{j=1}^{k}\left|\frac{\partial \varphi_{i}\left(x_{-1}^{*}\right)}{\partial x_{2 j}}\right|<\frac{1}{N-1}
$$

By lemma 4 there exists $\varepsilon>0$ such that $\varphi_{i}^{1}$ is a contraction on $\bar{U}_{i} \equiv \overline{\mathbb{B}}_{i}\left(x_{-1}^{*}, \varepsilon_{i}\right)$. By the proof of lemma $3 \varphi\left(x_{-1}\right)$ is a contraction on $\bar{U} \equiv \overline{\mathbb{B}}\left(x_{-1}^{*}, \varepsilon\right)$ where $\varepsilon \equiv \min \left\{\varepsilon_{i}\right\}$. But then by lemma $3 \phi$ is a contraction on $V \equiv \overline{\mathbb{B}}\left(x^{*}, \varepsilon\right)$.

I now present a numerical example that violates the condition of a dominant diagonal but satisfies the contraction condition (25). Suppose $k=N=2$ and $x^{*}$ is an interior symmetric equilibrium. Let the Jacobian $J\left(x^{*}\right)$ be given by

$$
J\left(x^{*}\right)=\left(\begin{array}{llll}
\Pi_{11}^{1} & \Pi_{12}^{1} & \Pi_{13}^{1} & \Pi_{14}^{1} \\
\Pi_{21}^{1} & \Pi_{22}^{1} & \Pi_{23}^{1} & \Pi_{24}^{1} \\
\Pi_{31}^{2} & \Pi_{32}^{2} & \Pi_{33}^{2} & \Pi_{34}^{2} \\
\Pi_{41}^{2} & \Pi_{42}^{2} & \Pi_{43}^{2} & \Pi_{44}^{2}
\end{array}\right)=\left(\begin{array}{cccc}
-1 & 1 / 2 & 1 / 2 & 0 \\
1 / 2 & -1 & 1 / 8 & 1 / 8 \\
0 & 1 / 2 & -1 & 1 / 2 \\
1 / 8 & 1 / 8 & 1 / 2 & -1
\end{array}\right)
$$

As can be seen from the first row diagonal dominance fails to hold. However, using the implicit function theorem the partial derivatives are $\frac{\partial}{\partial x_{21}} \varphi_{1}\left(x_{21}, x_{22}\right)=9 / 12, \frac{\partial}{\partial x_{22}} \varphi_{1}\left(x_{21}, x_{22}\right)=1 / 12$, $\frac{\partial}{\partial x_{21}} \varphi_{2}\left(x_{21}, x_{22}\right)=1 / 2$ and $\frac{\partial}{\partial x_{22}} \varphi_{2}\left(x_{21}, x_{22}\right)=1 / 6$. Hence $(25)$ is satisfied.

\section{References}

Alos-Ferrer, C. and Ania, A. B. (2005). The evolutionary stability of perfectly competitive behavior. Economic Theory, 26:497-516.

Amir, R., Jakubczyk, M., and Knauff, M. (2008). Symmetric versus asymmetric equilibria in symmetric supermodular games. International Journal of Game Theory, 37:307-320.

Dasgupta, P. and Maskin, E. (1986). The existence of equilibrium in discontinuous economic games, i: Theory. The Review of Economic Studies, 53(1):1-26. 
Dastidar, K. G. (2000). Is a unique cournot equilibrium locally stable? Games and Economic Behavior, 32:206-218.

Dixit, A. (1986). Comparative statics for oligopoly. International Economic Review, 27(1):107122.

Gale, D. and Nikaido, H. (1965). The jacobian matrix and global univalence of mappings. Math. Annalen, 159:81-93.

Gaudet, G. and Salant, S. W. (1991). Uniqueness of cournot equilibrium: New results from old methods. Review of Economic Studies, 58(2):399-404.

Grossman, G. M. and Shapiro, C. (1984). Informative advertising with differentiated products. Review of Economic Studies, 51:63-81.

Rosen, J. B. (1965). Existence and uniqueness of equilibrium points for concave n-person games. Econometrica, 33(3):520-534.

Salop, S. (1979). Monopolistic competition with outside goods. The Bell Journal of Economics, 10:141-156.

Simsek, A., Ozdaglar, A., and Acemoglu, D. (2007). Generalized poincare-hopf theorem for compact nonsmooth regions. Mathematics of operations research, 32(1):193-214.

Tang, A. K., Simsek, A., Ozdaglar, A., and Acemoglu, D. (2007). On the stability of p-matrices. Linear Algebra and its Applications, 426(1):22-32.

Topkis, D. M. (1998). Supermodularity and Complementarity. Princeton: University Press.

Vives, X. (1999). Oligopoly pricing. Cambridge: MIT Press.

Vives, X. (2005). Complementarities and games: New developments. Journal of Economic Literature, 43(2):437-479. 